\title{
Full-Field Surface Pressure Reconstruction Using the Virtual Fields Method
}

\author{
R. Kaufmann ${ }^{1}$ (D) B. Ganapathisubramani ${ }^{1} \cdot$ F. Pierron ${ }^{1}$
}

Received: 14 December 2018 / Accepted: 14 June 2019 / Published online: 24 July 2019

(C) The Author(s) 2019

\begin{abstract}
This work presents a methodology for reconstructing full-field surface pressure information from deflectometry measurements on a thin plate using the Virtual Fields Method (VFM). Low-amplitude mean pressure distributions of the order of few $\mathcal{O}(100) \mathrm{Pa}$ from an impinging air jet are investigated. These are commonly measured point-wise using arrays of pressure transducers, which require drilling holes into the specimen. In contrast, the approach presented here allows obtaining a large number of data points on the investigated specimen without impact on surface properties and flow. Deflectometry provides full-field deformation data on the specimen surface with remarkably high sensitivity. The VFM allows extracting information from the full-field data using the principle of virtual work. A finite element model is employed in combination with artificial grid deformation to assess the uncertainty of the pressure reconstructions. Both experimental and model data are presented and compared to show capabilities and restrictions of this method.
\end{abstract}

Keywords Deflectometry · Virtual Fields Method - Surface pressure reconstruction · Full-field measurement .

Fluid-structure interaction

\section{Introduction}

Full-field surface pressure measurements are highly relevant for engineering applications like material testing, component design in aerodynamics and the use of impinging jets for cooling, de-icing and drying. Surface pressure information can be used to determine aerodynamic loads [1] and to evaluate the performance of impinging jets used for heat and mass transfer [2]. They are however difficult to achieve, as available methods are not universally applicable. Most commonly, large numbers of pressure transducers are fitted into the investigated surface. This is an invasive technique as it requires one to drill holes into the sample. Further, it yields limited spatial resolution [3, 4]. Pressure sensitive paints allow obtaining full-field data, but are not suited for low-range differential pressure measurements [5, chapter $4.4 ; 6]$. They further require extensive calibration efforts, as well as a controlled experimental environment. Calculating

R. Kaufmann

rene.kaufmann@southampton.ac.uk

1 University of Southampton, Highfield, Southampton SO17 1BJ, UK pressure from Particle Image Velocimetry (PIV) is a noninvasive method that yields full-field data in the flow field $[7,8]$. This allows estimations of pressure along lines on which the surface coincides with the field of view.

Another approach is the reconstruction of pressure information from full-field surface deformation measurements by solving the local equilibrium equations. Recently, wall pressure was calculated from 3D-Digital Image Correlation (DIC) measurements on a flexible Kevlar wind-tunnel wall in an anechoic chamber [9]. This was achieved by projecting the measured deflections onto polynomial basis functions and inserting their derivatives into the corresponding equilibrium equations. The obtained pressure coefficients compared well to transducer data for the relatively large spatial scales that were investigated. Many problems in the field of fluid-structure interactions can be simplified to low amplitude loads acting on thin plates. This allows employing the Love-Kirchhoff thin plate theory [10] to write the local equilibrium of the plate. The required fullfield deformation information on the test surface can be obtained using a number of measurement techniques, e.g. DIC, Laser Doppler Vibrometers (LDV) or interferometry techniques. However, the fourth order deflection derivatives required to solve the Love-Kirchhoff equilibrium equation make an application in the presence of experimental noise 
challenging, particularly for low signal-to-noise ratios. To a degree, this issue can be addressed by applying regularisation techniques. In studies based on solving the equilibrium equation locally by employing a finite difference scheme, regularisation was achieved by applying wave number filters [11] or by adapting the number of data points used for the finite differences [12]. This allowed an identification and localisation of external vibration sources acting on the investigated specimen. Similarly, the acoustic component of a flow was identified using wave number filters in an investigation of a turbulent boundary layer [13]. Generally, the accuracy of this approach in terms of localisation and amplitude identification depends strongly on the chosen regularisation.

An alternative for solving the thin plate problem using full-field data is the Virtual Fields Method (VFM), which is based on the principle of virtual work and only requires second order deflection derivatives. The VFM is an inverse method that uses full-field kinematic measurements to identify mechanical material properties from known loading or vice versa. A detailed overview of the method and the range of applications is given in [14]. It notably does not require detailed knowledge of the boundary conditions and does not rely on computationally expensive iterative procedures. A study comparing Finite Element Model Updating, the Constitutive Equation Gap Method and the VFM for constitutive mechanical models using full-field measurements found that the VFM consistently performed best in terms of computational cost with reasonable results [15]. The VFM has been adapted for load reconstruction in a number of studies, including dynamic load identification in a Hopkinson bar $[16,17]$. The data were found to compare reasonably well to standard measurement techniques. The VFM was also used to reconstruct spatially-averaged sound pressure levels from an acoustic field using a scanning Laser Doppler Vibrometer (LDV) [18]. Dynamic transverse loads, as well as vibrations caused by acoustic pressure were identified using the same technique in [19]. The results were found to be accurate for distributed loads. The latter used a VFM approach based on piecewise virtual fields, which allows more accurate descriptions of boundary conditions for complex shapes and heterogeneous materials [20]. This approach was extended to random spatial wall pressure excitations in [21], reconstructing power spectral density functions from measured data and using the VFM to describe the plate response. The authors found that this method requires piecewise virtual fields to be defined over small regions. Recently, the VFM approach was combined with deflectometry for the identification of mechanical point loads of several $\mathcal{O}(1) \mathrm{N}$ [22]. Deflectometry is a highly sensitive technique for slope measurement [23]. It was successfully used in a range of applications like damage detection of composites [24], the analysis of stiffness and damping parameters of vibrating plates [25] and for imaging of ultrasonic lamb waves [26]. Since deflectometry measurements yield surface slopes, the combination with the VFM reduces the required order of derivatives of experimental data for pressure reconstruction to one. Known loads were reconstructed in [22] with good accuracy for certain reconstruction window sizes which were found empirically. Deflectometry and the VFM were also used to identify pressure auto-spectra of spatially averaged random excitations in [27]. The results agreed well with microphone array measurements, except at the structural resonance frequencies and for poor signal-to-noise-ratios. In the same study, the VFM approach was extended to membranes and the applicability was investigated using a simulated experiment. A shortcoming of these previous studies was that the accuracy was not assessed for unknown input loads. This is an important step because neither the resolution in space nor the uncertainty in pressure amplitude can be predicted directly as they depend on the signal amplitude and distribution, the noise level and the reconstruction parameters.

The main focus of the work presented here is the determination of static low-amplitude pressure distributions with peak values of few $\mathcal{O}(100) \mathrm{Pa}$ from time-averaged full-field slope measurements, as well as an assessment of the uncertainties of the method. In the following sections "Theory" and "Experimental Methods", a brief overview of the theoretical background and experimental setup is given. In "Experimental Results", experimental results are presented for two different specimen and for several reconstruction parameters. The pressure reconstructions are compared to pressure transducer measurements. Section "Simulated Experiments" introduces a numerical model for simulated experiments. This allows an assessment of the uncertainty of the method in terms of both systematic errors and the influence of random noise. In "Simulated Experiments", a finite element updating procedure is proposed to compensate for systematic errors.

\section{Theory}

\section{Impinging Jets}

A fan-driven, round air jet was used to apply a load on the specimen. The flow generated by this impinging jet can be divided into the free jet, stagnation and wall region [28]. These regions, shown in Fig. 1, consist of subregions with distinct flow features which are governed by the ratio between downstream distance and nozzle diameter H/D and Reynolds number Re. Directly downstream from the nozzle exit, the free jet develops for sufficiently large $H / D \gtrsim 2$ [29]. The velocity profile spreads as it moves 
Fig. 1 Impinging jet regions

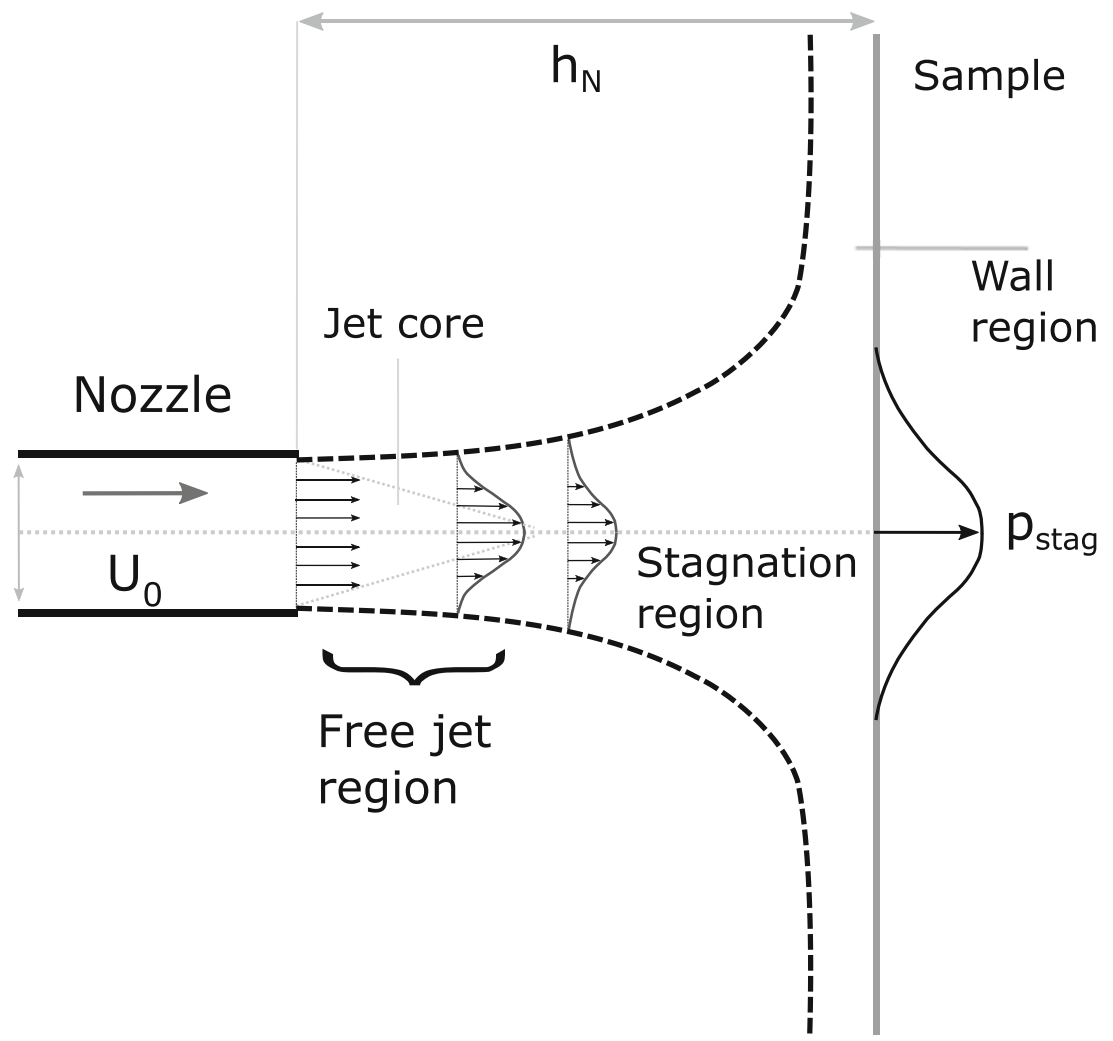

downstream due to entrainment and viscous diffusion causing a transfer of momentum to surrounding fluid particles. Upon approaching the impingement plate a stagnation region forms, characterized by an increase in static pressure up to the stagnation point on the plate surface. The rising static pressure results in pressure gradients diverting the flow radially away from the jet centerline. The laterally diverted flow forms the wall region. The pressure distribution on the impingement surface is approximately Gaussian [30]. This study focuses on the measurement of the mean load distribution on the impingement plate.

\section{Deflectometry}

Deflectometry is an optical full-field measurement technique for surface slopes [23]. Figure 2 shows a schematic of the setup. A camera measures the reflected image of a periodic spatial signal, here a cross-hatched grid, on the surface of a specular reflective sample. The distance between the grid and sample is denoted by $h_{\mathrm{G}}$ and the grid pitch by $p_{\mathrm{G}}$. The angle $\theta$ has to be sufficiently small to minimize grid distortion in the recorded image. A pixel directed at point $\mathrm{M}$ on the specimen surface will image the reflected grid at point $\mathrm{P}$ in an unloaded configuration. If a load is applied to the surface, it deforms locally and the same pixel will now image the reflected grid at point $\mathrm{P}^{\prime}$. It is assumed here that rigid body movements and out of plane deflections are negligible (for details see "Error Sources" below).
The displacement $\boldsymbol{u}$ between $\mathrm{P}$ and $\mathrm{P}^{\prime}$ relates to the phase difference $\mathrm{d} \phi$ in the grid signal in $\mathrm{x}$ - and $\mathrm{y}$-direction respectively as follows:

$d \phi_{x}=\frac{2 \pi}{p_{G}} u_{x}, d \phi_{y}=\frac{2 \pi}{p_{G}} u_{y}$

A spatial shift by one grid pitch $p_{\mathrm{G}}$ corresponds to a phase shift of $2 \pi$. However, a direct displacement estimation from the phase difference between a reference and a deformed

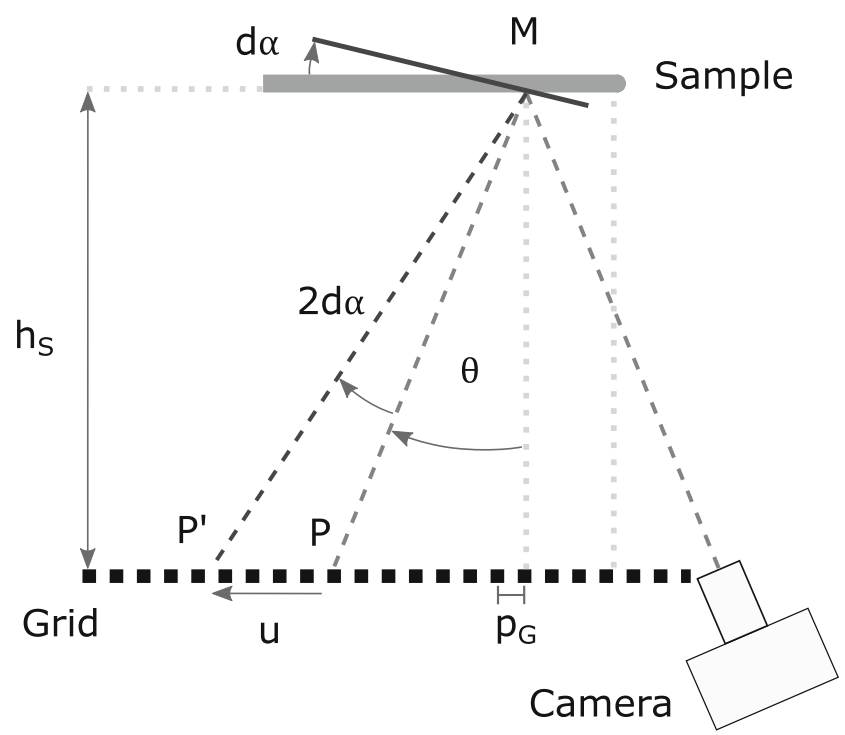

Fig. 2 Deflectometry setup, top view 
image does not take into account that the physical point on the plate surface is subject to a displacement. An iterative procedure to improve the displacement results given in [31, section 4.2] is employed here:

$\boldsymbol{u}_{n+1}(\boldsymbol{x})=-\frac{p_{G}}{2 \pi}\left(\phi_{\text {def }}\left(\boldsymbol{x}+\boldsymbol{u}_{n}(\boldsymbol{x})\right)-\phi_{r e f}(\boldsymbol{x})\right)$

A relationship between slopes and displacement is derived e.g. in [32]. It is based on geometric considerations and assumes that $\theta$ is sufficiently small, so the camera records images in normal incidence and $h_{\mathrm{G}}$ is large against the shift $\boldsymbol{u}$ :

$d \alpha_{x}=\frac{u_{x}}{2 h_{G}}, d \alpha_{y}=\frac{u_{y}}{2 h_{G}}$

Otherwise, a more complex calibration is required $[33,34]$. Equation (3) will be used here.

The spatial resolution of the method is driven by $p_{\mathrm{G}}$. The phase resolution is noise dependent and can be defined as the standard deviation of a phase map detected between two stationary images. Consequently, slope resolution depends on $p_{\mathrm{G}}, h_{\mathrm{G}}$ and the phase resolution.

\section{Phase detection}

The literature describes a number of methods for retrieving phase information from grid images, e.g., [31, 35, 36]. Here, a spatial phase-stepping algorithm is employed which allows investigating dynamic events $[37,38]$. One phase map is calculated per image. The chosen algorithm needs to be capable of coping with miscalibration, i.e. a slightly non-integer number of pixels per grid period. This can occur due to imperfections in the printed grid, misalignment between camera, sample and grid, lens distortion, as well as fill factor issues. In addition, the investigated signal is not generally sinusoidal. This requires an algorithm suppressing harmonics and sets a lower limit to the required number of samples, i.e. pixels recorded per grid pitch [39]. A windowed discrete Fourier transform algorithm using triangular weighting and a detection kernel size of two grid periods as used in e.g., [36] and [40] will be used in this study.

\section{Pressure Reconstruction}

The problem investigated here is a thin plate in pure bending, which allows the Love-Kirchhoff theory to be employed [41]. Assuming that the plate material is linear elastic, isotropic and homogeneous, the principle of virtual work is expressed by:

$$
\begin{aligned}
\int_{S} p w^{*} d S= & D_{x x} \int_{S}\left(\kappa_{x x} \kappa_{x x}^{*}+\kappa_{y y} \kappa_{y y}^{*}+2 \kappa_{x y} \kappa_{x y}^{*}\right) d S \\
& +D_{x y} \int_{S}\left(\kappa_{x x} \kappa_{y y}^{*}+\kappa_{y y} \kappa_{x x}^{*}-2 \kappa_{x y} \kappa_{x y}^{*}\right) d S \\
& +\rho t_{S} \int_{S} a w^{*} d S .
\end{aligned}
$$

$\mathrm{S}$ is the surface area, $\mathrm{p}$ the investigated pressure, $D_{x x}$ and $D_{x y}$ the plate bending stiffness matrix components, $\kappa$ the curvatures, $\rho$ the plate material density, $t_{S}$ the plate thickness, $a$ the acceleration, $w^{*}$ the virtual deflection and $\kappa^{*}$ the virtual curvatures. Here, the parameters $D_{x x}, D_{x y}$, $\rho$ and $t_{S}$ are known from the plate manufacturer. $\kappa$ and $a$ are obtained from deflectometry measurements, see "Data Acquisition and Processing" below. For the selection of the virtual fields $w^{*}$ and $\kappa^{*}$ one needs to take into account theoretical as well as practical restrictions of the problem like continuity, boundary conditions and sensitivity to noise.

The problem can be simplified by assuming the pressure $\mathrm{p}$ to be constant over the investigated area and by approximating the integrals with discrete sums.

$$
\begin{gathered}
p=\left(D_{x x} \sum_{i=1}^{N} \kappa_{x x}^{i} \kappa_{x x}^{* i}+\kappa_{y y}^{i} \kappa_{y y}^{* i}+2 \kappa_{x y}^{i} \kappa_{x y}^{* i}\right. \\
+D_{x y} \sum_{i=1}^{N} \kappa_{x x}^{i} \kappa_{y y}^{* i}+\kappa_{y y}^{i} \kappa_{x x}^{* i}-2 \kappa_{x y}^{i} \kappa_{x y}^{* i} \\
\left.+\rho t_{S} \sum_{i=1}^{N} a^{i} w^{* i}\right)\left(\sum_{i=1}^{N} w^{* i}\right)^{-1} .
\end{gathered}
$$

Here, $\mathrm{N}$ is the number of discretised surface elements $\mathrm{d} \mathrm{S}^{i}$.

\section{Virtual Fields}

For the present problem of identifying an unknown load distribution, it is beneficial to choose piecewise virtual fields due to their flexibility [18-20, 22]. In this study, the virtual fields are defined over a window of chosen size which is then shifted over the surface $\mathrm{S}$ until the entire area is covered. One pressure value is calculated for each window. In the following, this window will be referred to as pressure reconstruction window PRW. This procedure also allows for oversampling in the spatial reconstruction by shifting the window by less than a full window size.

Here, the only theoretical requirements for the virtual fields are continuity and differentiability. Since curvatures relate to deflections through their second spatial derivatives for a thin plate in pure bending, the virtual deflections are required to be $\mathrm{C}^{1}$ continuous. It is further necessary to eliminate the unknown contributions of virtual work along the plate boundaries. This is achieved by choosing virtual displacements and slopes that are zero around the window borders. 4-node Hermite 16 element shape functions as used in FEM [42] fulfill these requirements. The full equations defining these functions can be found in [14, chapter 14]. Figure 3 shows example virtual fields. 9 nodes are defined for a PRW. All degrees of freedom are set to zero except for the virtual deflection of the center node, which is set to 1 . 


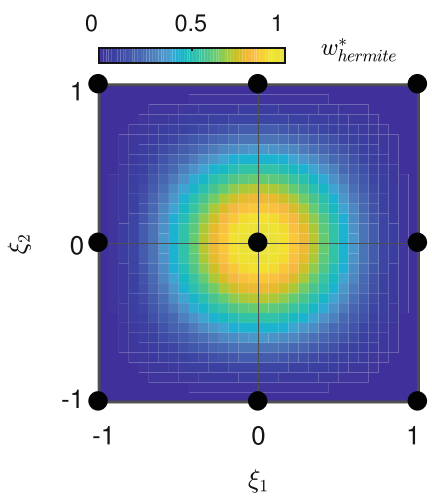

(a) Virtual displacements $w^{*}$

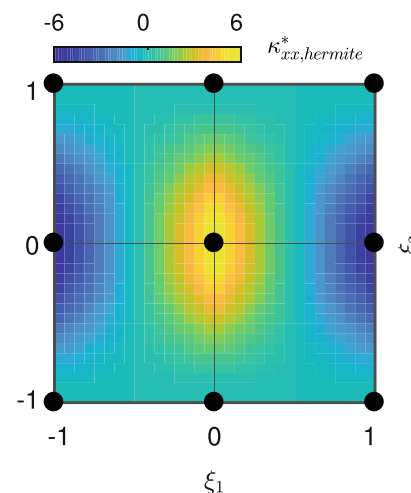

(b) Virtual curvatures $k_{x x}^{*}$

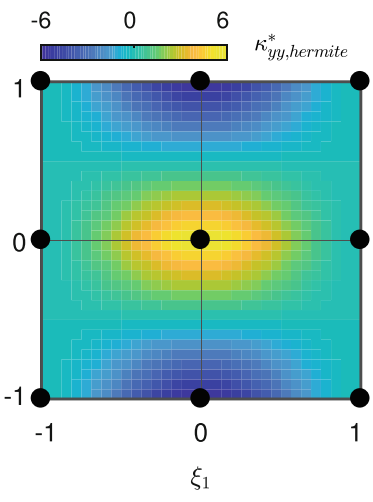

(c) Virtual curvatures $k_{y y}^{*}$

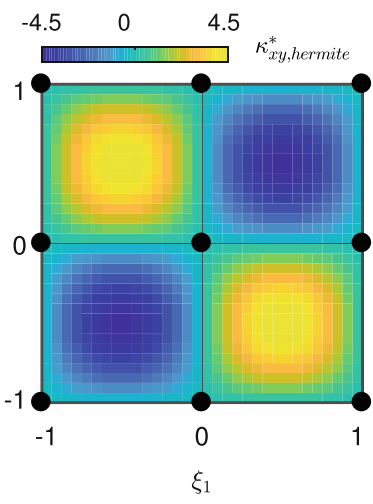

(d) Virtual curvatures $k_{x y}^{*}$

Fig. 3 Example Hermite 16 virtual fields with superimposed virtual elements and nodes (black). $\xi_{1}, \xi_{2}$ are parametric coordinates. The example window size is 32 points in each direction. Full equations can be found in [14, chapter 14]

The size of the PRW is an important parameter for the pressure reconstruction. Generally, the presence of random noise requires a larger PRW in order to average out the effect of noise on the pressure value within the window. A smaller PRW however can perform better at capturing small scale spatial structures, as large windows may average out amplitude peaks. One challenge in varying the window size is that the systematic error varies with it, as well as the effect of random noise on pressure reconstruction. This problem is investigated numerically in "Simulated Experiments".

\section{Experimental Methods}

\section{Setup}

Figure 4 shows a schematic of the experimental setup. A round, fan-driven impinging air jet was used to apply pressure on the specimen. The jet was fully turbulent at a downstream distance of $0.5 \mathrm{~cm}$ from the nozzle exit. The specimen was glued on a square acrylic frame. The grid was printed on transparency and fixed between two glass plates in the setup. A white light source was placed behind it. The camera was placed next to the grid at the same distance from the sample such that the reflected grid image is recorded at normal incidence. The distance between the sample and grid was chosen to be as large as possible in order to minimise the angle $\theta$ (see Fig. 2). Two different glass sample plates were investigated, one with thickness of $1 \mathrm{~mm}$ and the other $3 \mathrm{~mm}$. All relevant experimental parameters are listed in Table 1.

\section{Grid}

A cross-hatched grid printed on a transparency was used as the spatial carrier. Sine grids printed in $\mathrm{x}-$ and $\mathrm{y}$ direction would be preferable for phase detection as they do not induce high frequency harmonics in the phase detection. Printing these in sufficient quality is however difficult to achieve with standard printers. Using a hatched grid and slightly defocusing the image achieves a similar result because the discrete black and white areas become blurred, effectively yielding a grey scale transition between minimum and maximum intensity. This does however result in a slightly lower signal to noise ratio. It should be noted that when printing the grid, an integer number of printed

Fig. 4 Experimental setup

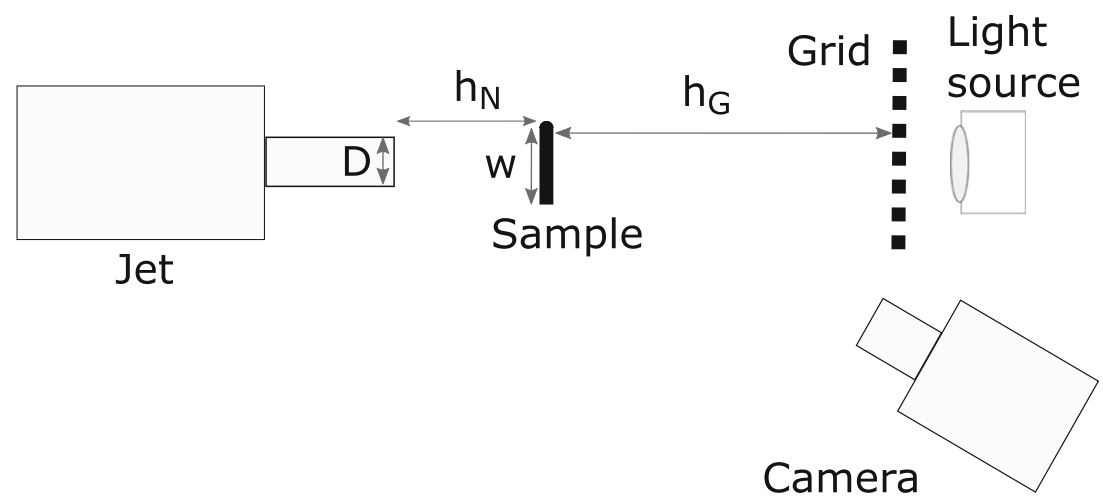


Table 1 Setup parameters

\begin{tabular}{|c|c|c|}
\hline \multicolumn{3}{|l|}{ Optics } \\
\hline \multirow{2}{*}{\multicolumn{2}{|c|}{ Camera }} & Photron Fastcam \\
\hline & & SA1.1 \\
\hline \multicolumn{2}{|l|}{ Technology } & CMOS \\
\hline \multicolumn{2}{|l|}{ Camera pixel size } & $20 \mu \mathrm{m}$ \\
\hline \multicolumn{2}{|l|}{ Surface fill factor } & $52 \%$ \\
\hline \multicolumn{2}{|l|}{ Dynamic range } & 12 bit \\
\hline \multicolumn{3}{|l|}{ Settings } \\
\hline \multicolumn{2}{|l|}{ Resolution } & $1024 \times 1024$ pixels \\
\hline Frame rate & $\mathrm{f}$ & $50 \mathrm{fps}$ \\
\hline \multicolumn{2}{|l|}{ Exposure } & $1 / 100 \mathrm{~s}$ \\
\hline \multicolumn{2}{|l|}{ Region of interest } & $64 \times 64 \mathrm{~mm}^{2}$ \\
\hline Magnification & M & 0.32 \\
\hline f-number & $N_{\text {Lens }}$ & 32 \\
\hline Focal length & $f_{\text {Lens }}$ & $300 \mathrm{~mm}$ \\
\hline \multicolumn{2}{|l|}{ Light source } & Halogen, $500 \mathrm{~W}$ \\
\hline \multicolumn{3}{|l|}{ Sample } \\
\hline \multicolumn{2}{|l|}{ Type } & First-surface mirror \\
\hline \multicolumn{2}{|l|}{ Material } & Glass \\
\hline Young's modulus & $E$ & $74 \mathrm{GPa}$ \\
\hline Poisson's ratio & $v$ & 0.23 \\
\hline Density & $\rho$ & $2.510^{3} \mathrm{~kg} \mathrm{~m}^{-3}$ \\
\hline Thickness & $t_{\mathrm{S}}$ & $1 \mathrm{~mm}, 3 \mathrm{~mm}$ \\
\hline Side length & $l_{\mathrm{s}}$ & ca. $90 \mathrm{~mm}, 190 \mathrm{~mm}$ \\
\hline \multicolumn{3}{|l|}{ Grid } \\
\hline \multicolumn{2}{|l|}{ Printed grid pitch } & $1.02 \mathrm{~mm}$ \\
\hline \multicolumn{2}{|l|}{ Grid-sample distance } & $1.03 \mathrm{~m}$ \\
\hline \multicolumn{2}{|l|}{ Pixels per pitch } & 8 \\
\hline \multicolumn{3}{|l|}{ Jet } \\
\hline \multicolumn{2}{|l|}{ Nozzle shape } & Round \\
\hline Nozzle diameter & $\mathrm{D}$ & $20 \mathrm{~mm}$ \\
\hline \multicolumn{2}{|l|}{ Area contraction ratio } & 0.13 \\
\hline Nozzle exit dynamic pressure & $p_{\text {exit }}$ & $630 \mathrm{~Pa}$ \\
\hline Reynold's number & $R e$ & $4 \cdot 10^{4}$ \\
\hline Sample-nozzle distance & $h_{\mathrm{N}}$ & $40 \mathrm{~mm}$ \\
\hline
\end{tabular}

dots per half pitch is required to avoid aliasing (e.g., [43]). For the current setup, grids with $1 \mathrm{~mm}$ pitch were printed on transparencies using a Konica Minolta bizhub C652 printers at $600 \mathrm{dpi}$.

\section{Sample}

The choice of the sample plate material and finish proved crucial for the investigation of small pressure amplitudes and spatial scales. The surface slopes under loading need to be large enough for detection, while at the same time the sample surface has to be plane enough for the grid image to be sufficiently in focus over the entire field of view. Perspex mirrors, polished aluminium and glass plates with reflective foils proved either too diffusive due to the Rayleigh criterion or insufficiently plane, resulting in a lack of depth of field when trying to image the reflected grid. Optical glass mirrors were chosen instead, as they provide adequate stiffness parameters and remain sufficiently plane when mounted. As it was possible to estimate the slope resolution from the noise level observed when recording two undeformed images on any sample thickness, deformation estimations based on the expected experimental load were used as input for finite element simulations to select suitable plate parameters. It was found that plates with thickness of $3 \mathrm{~mm}$ or lower were required. Good results were achieved using a $1 \mathrm{~mm}$ thick first-surface glass mirror as specimen. Still, fitting the $1 \mathrm{~mm}$ glass mirror on the frame caused it to bend slightly, resulting in small deviations from a perfect plane and subsequent local lack of depth of field. This was addressed by closing the aperture. A second, $3 \mathrm{~mm}$ thick mirror was used for comparison as it did not bend notably when mounted, though signal amplitudes for this case proved to be very low. The sample plates were glued onto a perspex frame along all edges.

\section{Transducer Measurements}

Pressure transducer measurements allowed a validation of the pressure reconstructions from deflectometry and the VFM. Endevco 8507C-2 type transducers were fitted in an aluminium plate along a line from the stagnation point outwards. The transducers have a diameter of $2.5 \mathrm{~mm}$ and were fitted with a spacing of $5 \mathrm{~mm}$. They were fitted to be flush with the surface to within approximately $0.5 \mathrm{~mm}$. Data was acquired at $10 \mathrm{kHz}$ over $20 \mathrm{~s}$ using a NI PXIe-4330 module.

\section{Data Acquisition and Processing}

One reference image was taken in an unloaded configuration before activating the jet. The jet required approximately $20 \mathrm{~s}$ to settle, after which a series of images was recorded. One data point was calculated per grid pitch during phase detection. Slopes were calculated relative to the reference image. Time averaged mean slope maps were calculated over $\mathrm{N}=5400$ measurements at $50 \mathrm{~Hz}$, limited by camera storage. From the slope maps the curvatures were obtained through spatial differentiation using centered finite differences. This requires knowledge of the physical distance between two data points on the specimen. It corresponds to the portion of the mirror required to observe the reflection of one grid pitch, which can be determined geometrically assuming $\theta$ is sufficiently small (see Fig. 2). In the present setup, camera sensor and grid were at the 
same distance from the mirror $h_{\mathrm{G}}$, such that the distance was half a printed grid pitch. Since differentiation tends to amplify the effect of noise, it can be beneficial to filter slope data before calculating curvatures. Here, the mean slopes were filtered using a 2D Gaussian filter, performing a convolution in the spatial domain. The filter kernel is characterized by its side length which is determined by the standard deviation, here denoted $\sigma_{\alpha}$, and truncated at $3 \sigma_{\alpha}$ in both directions. Because of its size, the filter kernel cannot be applied to the data points at the border of the field of view without padding. As padding should be avoided to prevent bias, $6 \sigma_{\alpha}-1$ data points were cropped along the edges of the field of view. While acting as a low-pass filter which reduces the effect of random noise, this technique also tends to reduce signal amplitude.

For the investigated problem of a mean flow profile, the accelerations average out to zero. This was confirmed with vibrometer measurements on several points along the test surface using a Polytec PDV 100 Portable Digital Vibrometer. Data was acquired at $4 \mathrm{kHz}$ over $20 \mathrm{~s}$. The noise level in LDV measurements was $0.3 \mathrm{~m} \mathrm{~s}^{-2}$. The observed standard deviations varied with the position along the plate surface and reached up to $1.4 \mathrm{~m} \mathrm{~s}^{-2}$. Therefore, the term involving accelerations in Eq. 4 is zero as well and will therefore be neglected in the following.

Pressure reconstructions were conducted for several PRW sizes. The results were oversampled by shifting the PRW over the investigated field of view by one data point per iteration. Note that due to the finite size of these windows, half a PRW of data points is lost around the edges of the field of view.

\section{Experimental Results}

Slope maps obtained from deflectometry measurements were processed and temporally averaged as described in "Data Acquisition and Processing". Results for both specimens are presented in the following, one plate with $1 \mathrm{~mm}$ thickness and $90 \mathrm{~mm}$ side length, and one with $3 \mathrm{~mm}$ thickness and $190 \mathrm{~mm}$ side length. The region of interest is $64 \mathrm{~mm}$ in both directions for each test cases. Figure 5(a)-(d) show the measured mean slope maps for both test plates. Distances are given in terms of radial distance from the impinging jet's stagnation point $r$, normalized by the nozzle diameter $D$, in $\mathrm{x}$ - and y-direction respectively. Note that the region of interest showing the jet center does not coincide with the plate center, so the slope amplitudes are not necessarily symmetric. The signal amplitudes for the $3 \mathrm{~mm}$ test case are significantly lower than for the $1 \mathrm{~mm}$ case. Slope shapes are different for both cases because the plates have different side length while the field of view remains the same size. Further, the stagnation point is off-center in the $3 \mathrm{~mm}$ test.

Figure 5(e)-(p) shows mean curvature maps with and without Gaussian filter. Stripes are visible in all curvature maps for the unfiltered $1 \mathrm{~mm}$ test data. This indicates the presence of a systematic error source in the experimental setup. Without slope filter, curvatures obtained from the $3 \mathrm{~mm}$ plate test are governed by noise. The curvature map for $\bar{\kappa}_{x x}$ (Fig. $5(\mathrm{~g})$ ) additionally shows fringes. These disappear after slope filtering, though filtered data still appear asymmetric, again indicating a systematic error. To assure that this issue occurring in for both plates does not originate from a lack of convergence, mean and instantaneous curvature maps were calculated and compared. All maps show the same bias, with small variations in amplitude.

This may be caused by misalignment between grid and image sensor due to imperfections in the printed grid, combined with the CMOS chip's fill factor. This results in a slightly varying number of pixels per grid pitch over the field of view, which leads to errors in phase detection and fringes. While this issue could be mitigated by careful realignment of camera and grid as well as slightly defocusing the image to address the low camera fill factor, it could not be fully eliminated. Another possible error source is the deviation of the plate surface from a perfect plane, e.g. due to deformations of the sample during mounting. Since differentiation amplifies the impact of noise, filtering the slope maps yields much smoother curvature maps. The downside is a possible loss of signal amplitude and of data points along the edges (see "Data Acquisition and Processing").

Figure 6(a)-(d) show pressure reconstructions using different PRW sizes. Pressure is given in terms of difference to atmospheric pressure, $\Delta p$. Here, one data point corresponds to a physical distance of $0.5 \mathrm{~mm}$, such that a PRW of 28 points corresponds to a window side length of $14 \mathrm{~mm}$ or $0.7 r D^{-1}$. The large number of data points is a result of oversampling by shifting the PRW over the investigated area by one point per iteration. The expected Gaussian shape of the distribution is found to be well reconstructed for filtered data and sufficiently large PRW, here above ca. 22 data points, for the $1 \mathrm{~mm}$ plate. Reconstructions from $3 \mathrm{~mm}$ plate tests are less symmetric. The position of the stagnation point is visible for all shown parameter combinations, but the shape of the distribution shows a recurring pattern which stems from the systematic error already observed in curvature maps. For both tests, some reconstructions show areas of negative differential pressure, which is unexpected for the mean distributions in this flow. This is likely to be a consequence of random noise, as similar patterns were observed in simulated experiments for noisy model data (see "Grid Deformation Study" below). For comparisons with the transducer measurements, 


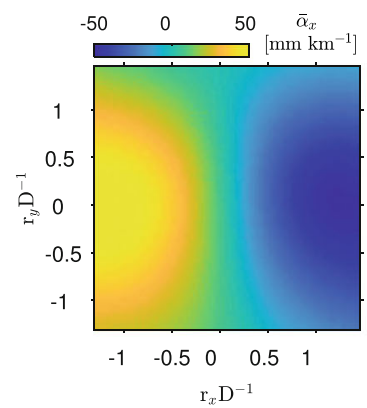

(a) $\bar{\alpha}_{x}$ for $t_{\mathrm{S}}=1 \mathrm{~mm}$ plate test.

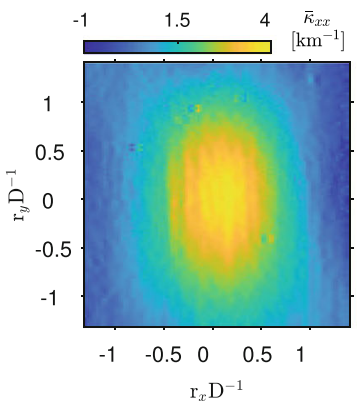

(e) $\bar{\kappa}_{x x}$ from unfiltered slopes for $t_{\mathrm{S}}=1 \mathrm{~mm}$ plate test.

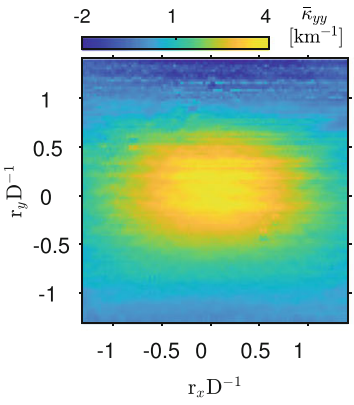

(i) $\bar{\kappa}_{y y}$ from unfiltered slopes for $t_{\mathrm{S}}=1 \mathrm{~mm}$ plate test.

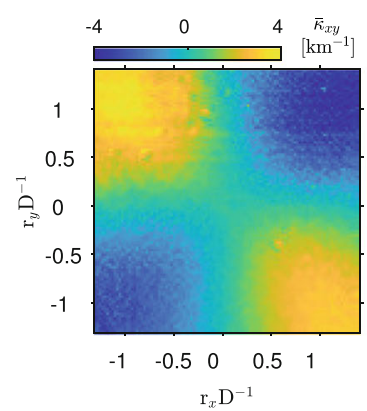

(m) $\bar{\kappa}_{x y}$ from unfiltered slopes for $t_{\mathrm{S}}=1 \mathrm{~mm}$ plate test.

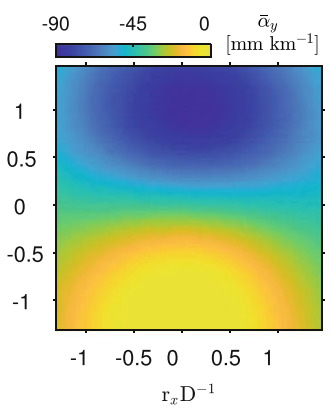

(b) $\bar{\alpha}_{y}$ for $t_{\mathrm{S}}=1 \mathrm{~mm}$ plate test.

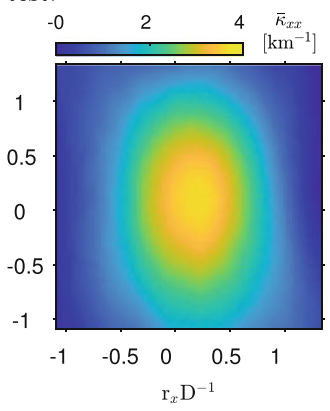

(f) $\bar{\kappa}_{x x}$ from filtered slopes for $t_{\mathrm{S}}=1 \mathrm{~mm}$ plate test, $\sigma_{\alpha}=3$.

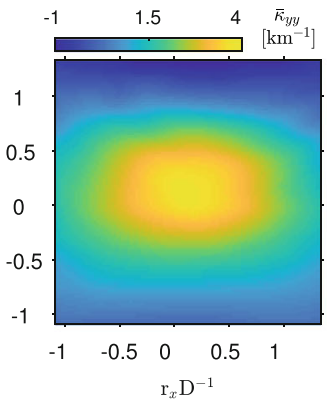

(j) $\bar{\kappa}_{y y}$ from filtered slopes, $\sigma_{\alpha}=3$ for $t_{\mathrm{S}}=1 \mathrm{~mm}$ plate test

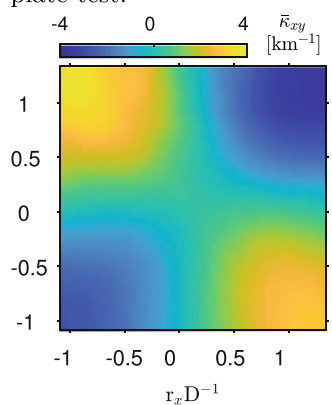

(n) $\bar{\kappa}_{x y}$ from filtered slopes for $t_{\mathrm{S}}=1 \mathrm{~mm}$ plate test, $\sigma_{\alpha}=3$.

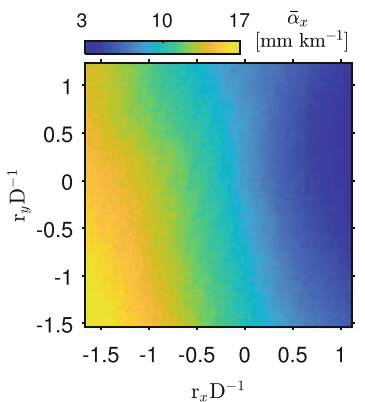

(c) $\bar{\alpha}_{x}$ for $t_{\mathrm{S}}=3 \mathrm{~mm}$ plate test.

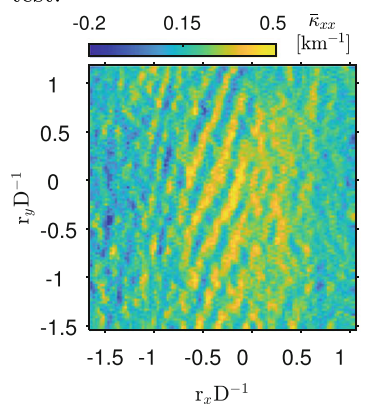

(g) $\bar{\kappa}_{x x}$ from unfiltered slopes for $t_{\mathrm{S}}=3 \mathrm{~mm}$ plate test.

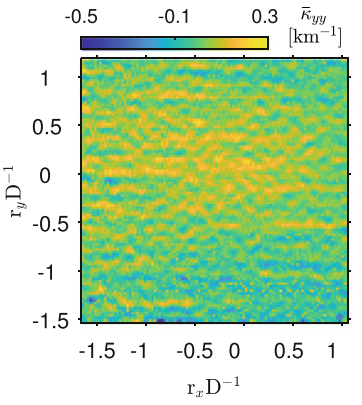

(k) $\bar{\kappa}_{y y}$ from unfiltered slopes for $t_{\mathrm{S}}=3 \mathrm{~mm}$ plate test.

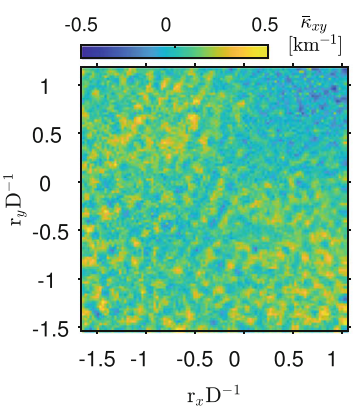

(o) $\bar{\kappa}_{x y}$ from unfiltered slopes for $t_{\mathrm{S}}=3 \mathrm{~mm}$ plate test.

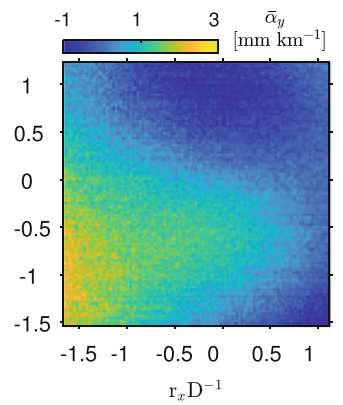

(d) $\bar{\alpha}_{y}$ for $t_{\mathrm{S}}=3 \mathrm{~mm}$ plate test.

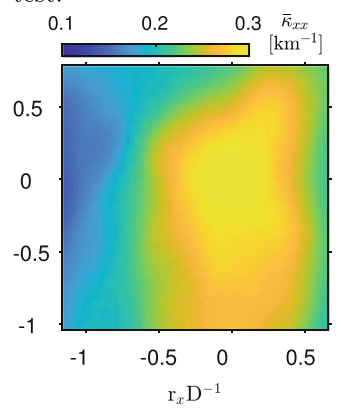

(h) $\bar{\kappa}_{x x}$ from filtered slopes for $t_{\mathrm{S}}=3 \mathrm{~mm}$ plate test, $\sigma_{\alpha}=6$.

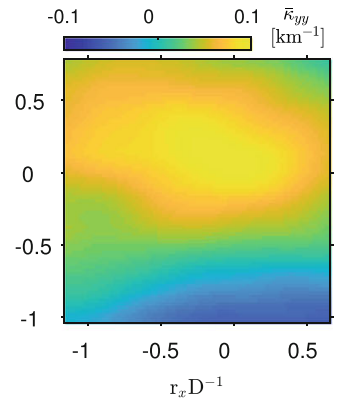

(l) $\bar{\kappa}_{y y}$ from filtered slopes for $t_{\mathrm{S}}=3 \mathrm{~mm}$ plate test, $\sigma_{\alpha}=6$.

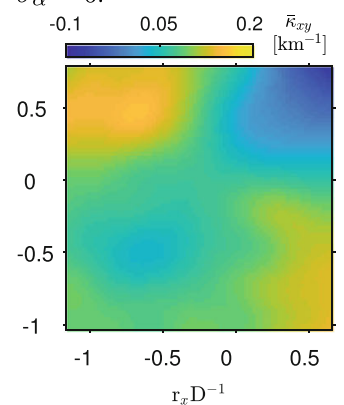

(p) $\bar{\kappa}_{x y}$ from filtered slopes for $t_{\mathrm{S}}=3 \mathrm{~mm}$ plate test, $\sigma_{\alpha}=6$.

Fig. 5 Measured mean slope and curvature maps

pressure reconstructions were averaged circumferentially for each corresponding radial distance from the stagnation point. Figure 6(e) and (f) show the results. The vertical error bars on transducer data represent both the systematic errors of the equipment as well as the random error of the mean pressure value. The horizontal error bars indicate the uncertainty in placing the transducers relative to the jet. Results from the $1 \mathrm{~mm}$ plate measurements appear to show 


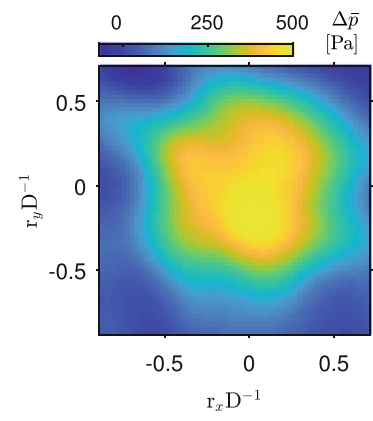

(a) $\Delta \bar{p}$ for $1 \mathrm{~mm}$ plate test, $\sigma_{\alpha}=3$, PRW $=28$.

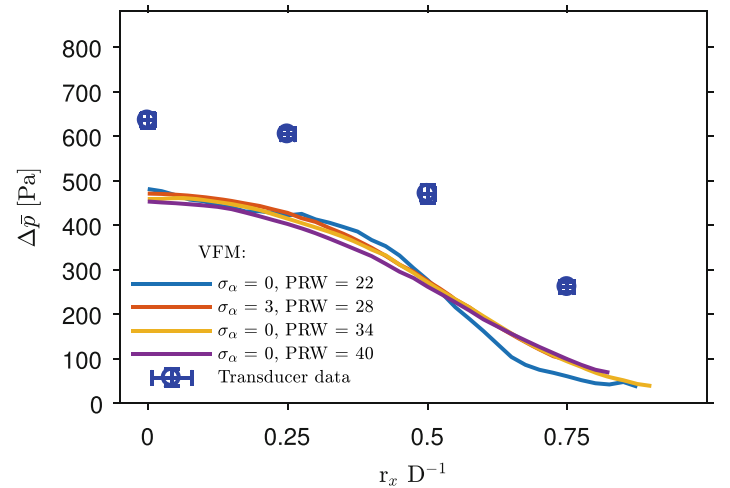

(e) Comparison between transducer measurements and VFM pressure reconstructions for $1 \mathrm{~mm}$ plate test.

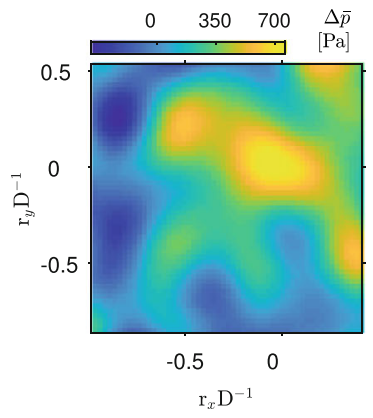

(c) $\Delta \bar{p}$ for $3 \mathrm{~mm}$ plate test, $\sigma_{\alpha}=6, \mathrm{PRW}=18$.

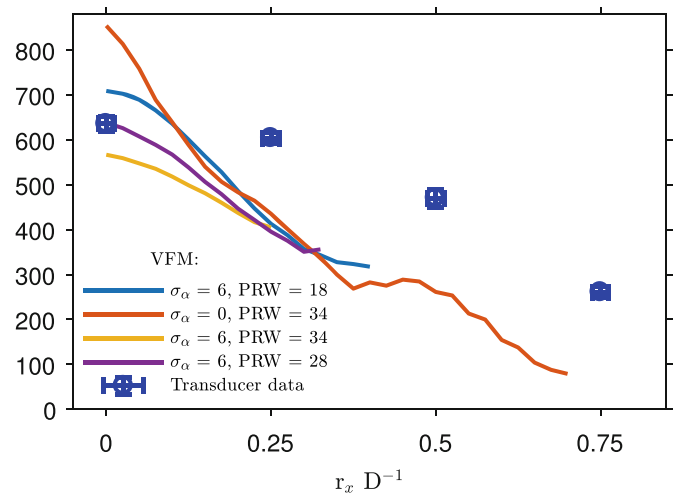

(f) Comparison between transducer measurements and VFM pressure reconstructions for $3 \mathrm{~mm}$ plate test.

Fig. 6 Comparison of VFM pressure reconstruction with pressure transducer data

a systematic underestimation of the pressure amplitude at all points. Possible sources for this error are discussed in detail in "Error Sources" below. However, the shape of the distribution is captured reasonably well. The $13 \mathrm{~mm}$ plate results show a good reconstruction of the peak amplitude, but the shape of the pressure distribution deviates due to the influence of random noise patterns. The results clearly show that the effects of the size of the PRW and the Gaussian smoothing kernel $\sigma_{\alpha}$ on the reconstruction outcome are significant. Therefore, the influence of the reconstruction parameters is investigated numerically in the following section.

\section{Simulated Experiments}

Comparisons of the VFM pressure reconstruction with the pressure transducer data shows that there are discrepancies between the results. Furthermore, it is unclear what parts of the reconstructed pressure amplitude stems from signal, random noise or systematic error. Processing experimental data with noise can produce pressure distributions that are indistinguishable from the signal of interest. It is also important to note that the complex measurement chain from images to pressure does not allow for analytical expressions to be obtained and only numerical simulations can shed light on the problem.

Numerical studies allow addressing this problem and estimating the effects of random and systematic error [31]. As a first step, a finite element model of the investigated thin plate problem is created. By applying a model load, the local displacements and slopes that result from the bending experiment can be simulated. For the next step, the grid image recorded with the camera is modelled numerically. The simulated displacements are used to calculate the deformations of the model grid image. Experimentally observed grey level noise is added to these grids. The simulated grids serve as input for a study of the influence of processing parameters on the pressure reconstruction. Comparisons with the model load allow an assessment of the uncertainties of the processing technique in the presence of random noise. In the last subsection, a finite element correction procedure is introduced to compensate for the reconstruction error.

\section{Finite Element Model}

Numerical data of slope maps from a thin plate bending under a given load distribution was calculated using a finite element simulation. This was conducted using the software 


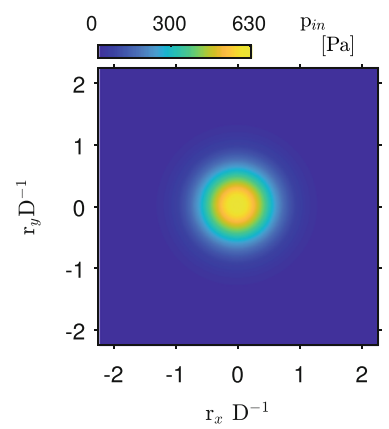

(a) Input load distribution.

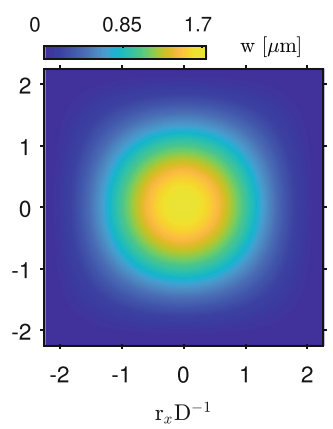

(b) Modelled deflections.

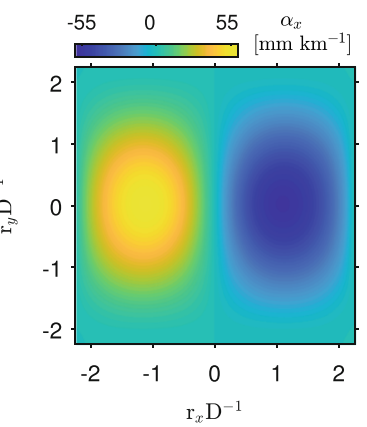

(c) Modelled slopes $\alpha_{x}$.

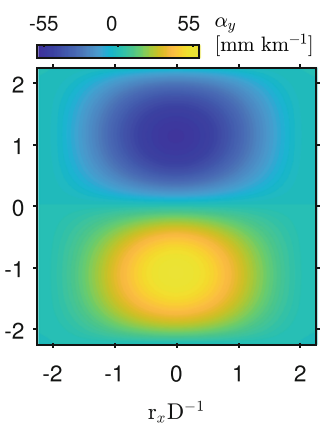

(d) Modelled slopes $\alpha_{y}$.

Fig. 7 ANSYS model in- and output for $1 \mathrm{~mm}$ plate model

ANSYS APDLv181. SHELL181 elements were chosen as they are well suited for modelling the investigated thin plate problem [44]. Both experimental test plates were simulated as homogeneous with the parameters detailed in Table 1 . All degrees of freedom were fixed along the edges. For both plates a square mesh was used with 1440 elements for the $1 \mathrm{~mm}$ thick plate and 2280 elements for the $3 \mathrm{~mm}$ thick plate. This allowed obtaining 1024 points in a window corresponding to $64 \mathrm{~mm}$, which corresponds to the experimental number of camera pixels and field of view. Figure 7(a) shows the Gaussian pressure distribution used as input, with an amplitude of $630 \mathrm{~Pa}$ and $\sigma_{\text {load }}=9 \mathrm{~mm}$. Figure 7(b) shows the resulting deflections, Fig. 7(c) and (d) the model slopes for the $1 \mathrm{~mm}$ plate case.

\section{Systematic Error}

The simulated slopes can be used as input for the VFM pressure reconstruction the same way as those obtained experimentally. This allows an assessment of the systematic error of the processing technique independent from experimental errors. A metric for estimating the error of a reconstruction was defined taking into account the difference between reconstructed and input pressure amplitude in terms of the local input amplitude at each point:

$\epsilon=\frac{1}{N} \sum_{i=1}^{N}\left|\sqrt{\left(\mathrm{p}_{\text {rec }, i}-\mathrm{p}_{i n, i}\right)^{2}} / \mathrm{p}_{i n, i}\right|$

$p_{\text {rec, } \mathrm{i}}$ is the reconstructed and $p_{\text {in, } \mathrm{i}}$ the input pressure at each point $i$ with a total number of points N. Pressure values below $1 \mathrm{~Pa}$ were omitted for this metric. Figure 8(a) shows the results for the accuracy estimate for pressure reconstructions from noise free slope data for different PRW. The results are oversampled as in the experimental case by shifting the PRW by one point per iteration. A minimum exists at PRW $=22$ with $\epsilon=0.12$, which indicates an average accuracy of ca. $88 \%$ of the local amplitude. The corresponding pressure reconstruction map is shown in Fig. 8(b). It should be noted that the local pressure amplitudes are underestimated for all investigated cases. For increasing PRW sizes, the peak amplitude is
Fig. 8 Systematic error estimate for VFM

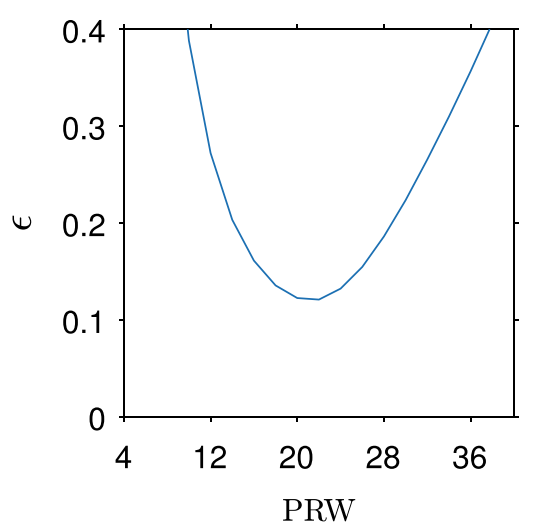

(a) Uncertainty estimate for varying PRW size.

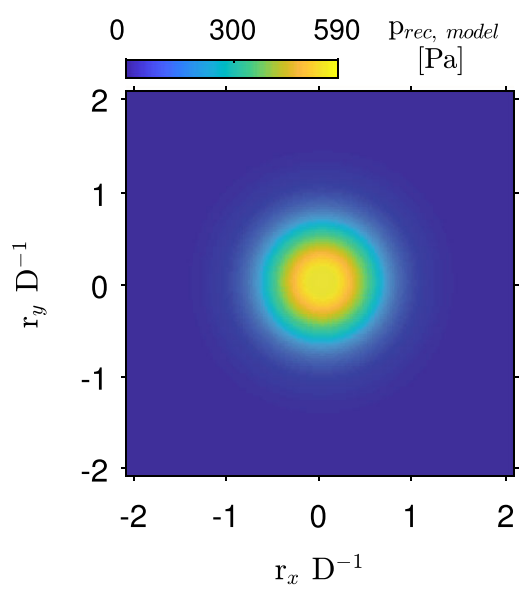

(b) Example pressure reconstruction from simulated slopes using PRW $=22$. 
underestimated because the virtual fields act as a weighted average over the entire window. Small PRWs were expected to yield best results in a noise free environment since they average over fewer data points. This is not confirmed here. Different finite element mesh sizes were tested to rule out model convergence issues. The low accuracy obtained for small windows is probably due to a lack of heterogeneity of (real) curvature in small windows. If curvatures are constant, they can be taken out of the integral in equation (4). Because the virtual curvatures average out to zero over one window, the integral then yields zero. For small windows, this situation is approached, likely leading to wrong pressure values. Choosing heterogeneous virtual curvature fields could be used to address this issue in future studies. One approach could be to defined more nodes on each virtual field and a non-zero virtual deflections on a node other than the center one to increase heterogeneity. Another way could be to employ higher order approaches for pressure calculation within one window, which is expected to yield higher accuracy for large PRW.

\section{Grid Deformation Study}

Artificial grid deformation allows for a more comprehensive assessment of error propagation by including the effects of camera resolution and noise. Following the approach described in [45], a periodic function with a wavelength corresponding to the experimental grid pitch was used in $\mathrm{x}$ and $y$-direction to generate the artificial grid.

$$
\begin{aligned}
I(x, y)= & I_{\min }+\frac{I_{\min }-I_{\max }}{2}+\frac{I_{\max }}{4} \\
& \cdot\left(\cos \left(\frac{2 \pi x}{p_{G}}\right)+\cos \left(\frac{2 \pi y}{p_{G}}\right)\right. \\
& \left.-\left|\cos \left(\frac{2 \pi x}{p_{G}}\right)-\cos \left(\frac{2 \pi y}{p_{G}}\right)\right|\right)
\end{aligned}
$$

Here, $I_{\min }$ and $I_{\max }$ are the minimum and maximum intensity values of the experimental grid images. The signal amplitude values were discretised to match the camera's dynamic range. All simulated image parameters were set to replicate the experimental conditions as described in Table 1. This spatial grid signal was oversampled by a factor of 10 and spatially integrated to simulate the signal recording process of the camera, as detailed in [45]. To further assess the actual experiment, random noise was added to the artificial grid images based on the grey level noise measured during experiments, here $0.95 \%$ and $0.61 \%$ of the used dynamic range in case of the $1 \mathrm{~mm}$ and $3 \mathrm{~mm}$ plate tests respectively. It varies because the illumination varied between both experiments, such that the used dynamic range was different. The amount of random noise is reduced with the number of measurements over which the mean value is calculated. However, the reduction of noise is not described by $1 / \sqrt{N}$ as would be expected. The same observation was made in [43]. It was investigated by taking a series of images without applying a load to the specimen. It was found that the amount of noise in phase maps increases with the time that has passed between two images being taken. It is likely that this is a result of small movements or deformations of the sample, printed grid and camera due to vibrations and temperature changes during the measurement. This does not fully account for the observed effect however. As a consequence, the amount of random noise for averages over multiple measurements has to be determined experimentally. For 5400 measurements on the undeformed sample, it was found that the random noise in phase was reduced by a factor of ca. 2.5 compared to two measurements. The values are statistically well converged after 30 realisations of simulated noise.

The simulation neglects the effects of grid defects, lens imperfections, inhomogeneous illumination and imperfections of the specimen. However, it does account for any systematic errors associated with the number of pixels on the camera sensor and the random errors coming from grey level noise in the images. Figure 9 shows a close-up view of simulated and experimental grid images. Simulated slopes yield corresponding deformations of the artificial grid at every point using equations (1) and (3). The obtained artificial grids for deformed and undeformed configurations can now be used as input for the phase detection algorithm. Areas with negative pressure amplitude were observed in reconstructions from noisy model data, very similar to those observed experimentally. A lower limit for pressure resolution was determined by adding noise to two undeformed artificial grids and processing them. The standard deviation of pressure values obtained from this reconstruction can be interpreted as a metric for the lower detection limit of the pressure reconstruction for the corresponding parameter combination. Values below the obtained threshold are neglected in all reconstructions in the following.

Phases obtained from artificial, deformed grids were processed and the reconstructed and input pressure were compared using the metric introduced in equation (6). This allows quantifying the systematic error of phase detection and VFM for all combinations of the relevant processing parameters. Oversampling in the phase detection algorithm, i.e. calculating more than one phase value per grid pitch, was found to improve the results, though at high computational cost. Particularly in combination with larger PRW and slope filter kernels, phase oversampled slope maps yield diminishing improvements in accuracy in terms of the overall cost. In the VFM pressure reconstruction, oversampling provided a significant improvement at acceptable cost. The slope filter kernel size $\sigma_{\alpha}$ also increases computational 
Fig. 9 Example grid sections

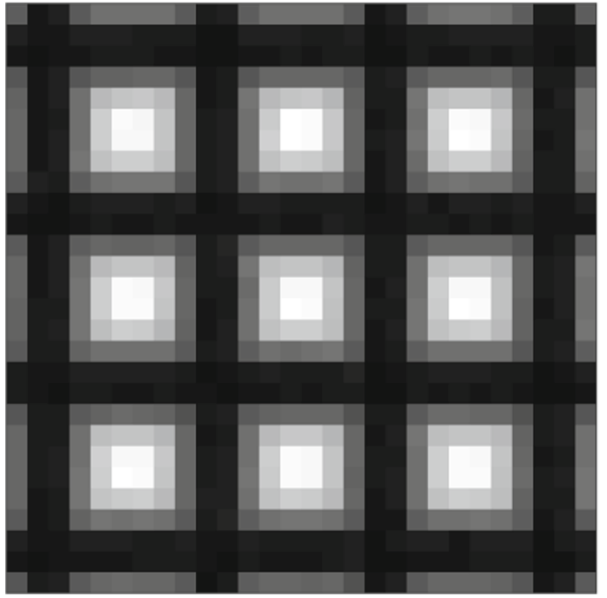

(a) Artificial grid after integration and with added noise.

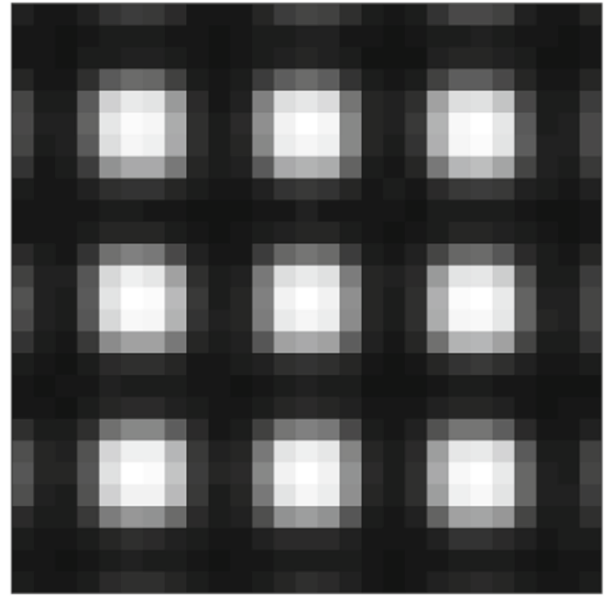

(b) Experimental grid. cost, but mitigates the effects of random noise efficiently. The influence of both the size of $\sigma_{\alpha}$ and PRW are investigated in the following as they yield the most significant improvements.

Figure 10(a) and (b) show the findings for varying parameters $\sigma_{\alpha}$ and PRW for each plate. These allow selecting parameter combinations with highest precision in terms of amplitude over the entire field of view (Fig. 11). Figures 12 and 13 show example comparisons of pressure reconstructions for different $\epsilon$. Figure 12 shows experimental data with two different parameter combinations for both plates and Fig. 13 below shows the corresponding results obtained using model data. For reference, Fig. 11 shows on top the model input distribution sections in the respective field of

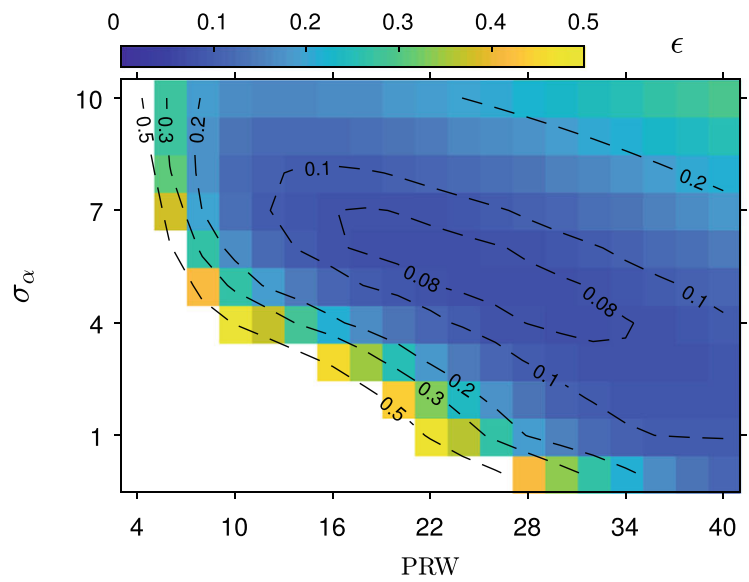

(a) Error estimates for varying slope filter kernel and PRW size for $1 \mathrm{~mm}$ plate test and with grey level noise $0.95 \%$ of the dynamic range. view. As expected, reconstructions using larger smoothing kernels tend to yield lower peak amplitudes. However, the amplitudes in other areas are be captured better, as noise induced peaks are filtered more efficiently. The fact that some numerical reconstructions do not represent Gaussian distributions well shows that noise effects are not averaged out entirely. For the low signal to noise ratio encountered in the $3 \mathrm{~mm}$ plate case, some reconstructions overestimate the peak pressure amplitude. This is a consequence of the differentiation of slope noise, which leads to large curvature and thus pressure values. Since this also leads to areas in which the pressure amplitude is underestimated, the effect averages out for sufficiently large slope smoothing kernel and PRW.

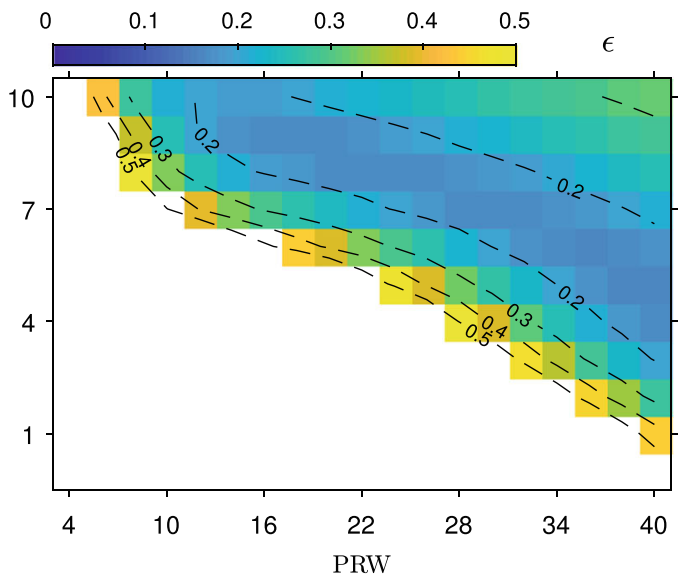

(b) Error estimates for varying slope filter kernel and PRW size for $3 \mathrm{~mm}$ plate test and with grey level noise $0.6 \%$ of the dynamic range.

Fig. 10 Pressure reconstruction accuracy analysis 


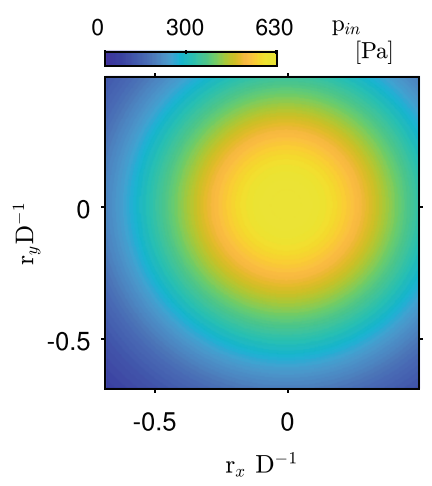

(a) Section corresponding to $\sigma_{\alpha}=6, \mathrm{PRW}=28$ reconstruction.

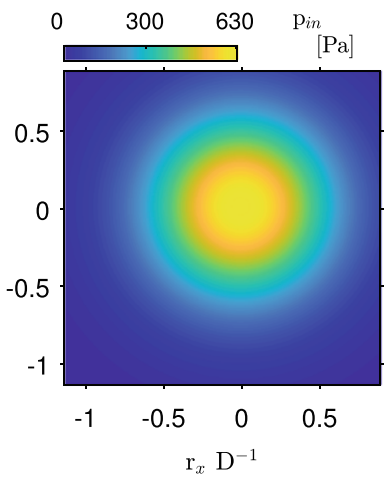

(b) Section corresponding to $\sigma_{\alpha}=0$, PRW $=22$ reconstruction.

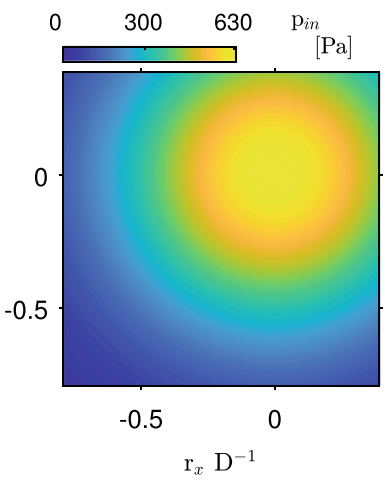

(c) Section corresponding to $\sigma_{\alpha}=6, \mathrm{PRW}=34$ reconstruction.

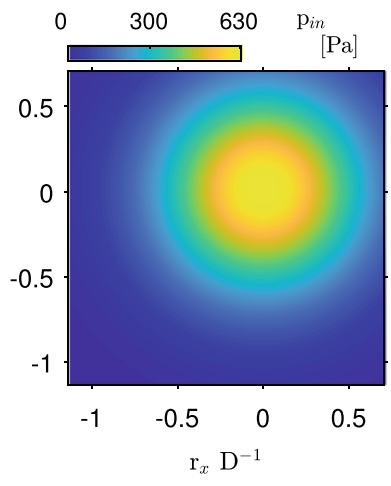

(d) Section corresponding to $\sigma_{\alpha}=2$, PRW $=28$ reconstruction.

Fig. 11 Model input pressure distribution sections for comparison with reconstruction results

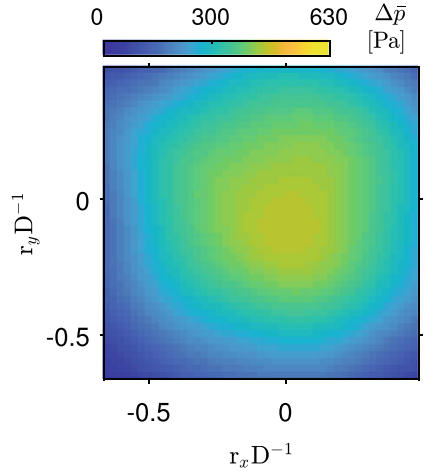

(a) Pressure reconstruction for $1 \mathrm{~mm}$ plate good accuracy estimate $\left(\sigma_{\alpha}=6\right.$, PRW $=28$, $\epsilon=0.08)$.

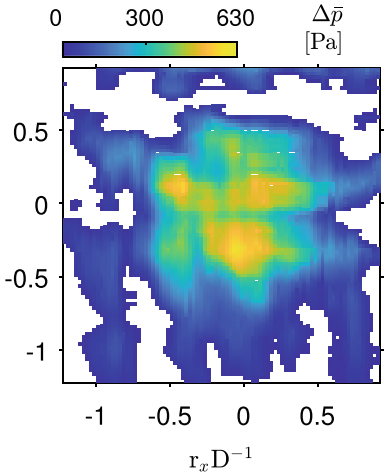

(b) Pressure reconstruction for $1 \mathrm{~mm}$ plate for $\sigma_{\alpha}=0$, $\mathrm{PRW}=22, \epsilon=0.4$.

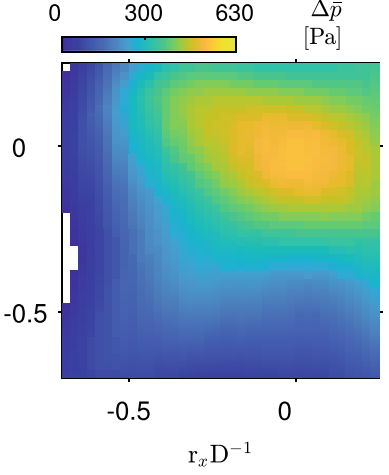

(c) Pressure reconstruction for $3 \mathrm{~mm}$ plate for good accuracy estimate $\left(\sigma_{\alpha}=6\right.$, $\mathrm{PRW}=34, \epsilon=0.2)$.

Fig. 12 Comparison of pressure reconstructions from experimental data for different parameter combinations

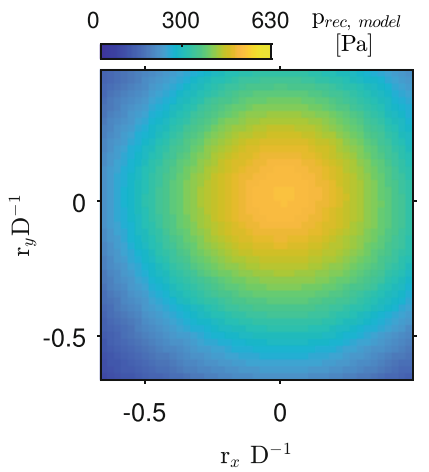

(a) Pressure reconstruction for $1 \mathrm{~mm}$ plate for good accuracy estimate $\left(\sigma_{\alpha}=6, \mathrm{PRW}=28\right.$, $\epsilon=0.08)$.

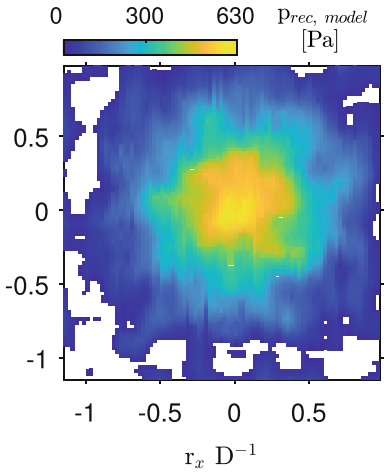

(b) Pressure reconstruction for $1 \mathrm{~mm}$ plate for poor accuracy estimate $\left(\sigma_{\alpha}=0\right.$, $\mathrm{PRW}=22, \epsilon=0.4$ )

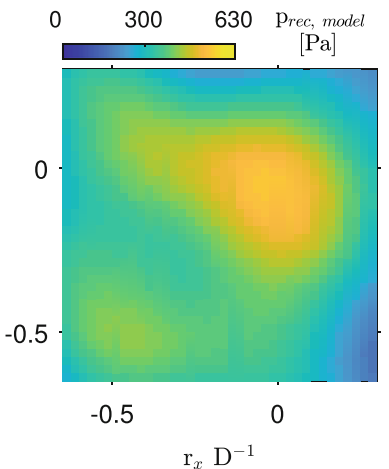

(c) Pressure reconstruction for $3 \mathrm{~mm}$ plate for good accuracy estimate $\left(\sigma_{\alpha}=6\right.$, $\mathrm{PRW}=34, \epsilon=0.2)$.

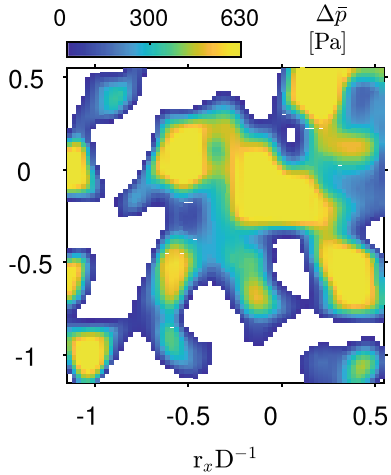

(d) Pressure reconstruction for $3 \mathrm{~mm}$ plate for poor accuracy estimate $\left(\sigma_{\alpha}=2\right.$, $\mathrm{PRW}=28, \epsilon>0.5)$.

Fig. 13 Comparison of pressure reconstructions from noisy model data for different parameter combinations

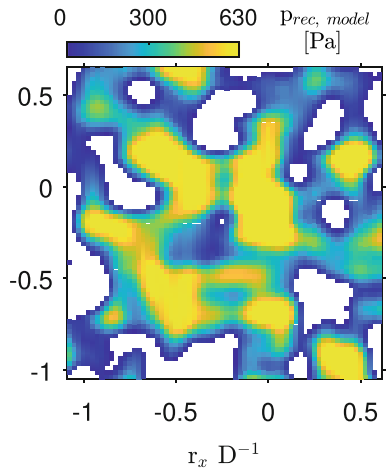

(d) Pressure reconstruction for $3 \mathrm{~mm}$ plate for poor accuracy estimate $\left(\sigma_{\alpha}=2\right.$, $\mathrm{PRW}=28, \epsilon>0.5)$. 


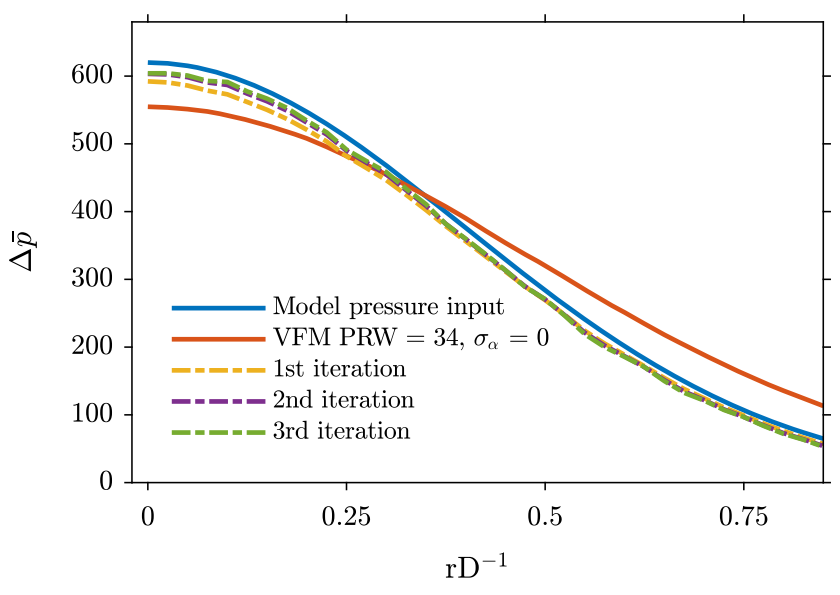

Fig. 14 FE corrected results for noise free model data

\section{Finite Element Correction}

The systematic error caused by the reconstruction technique which was identified above shows an underestimation of the input pressure for noise free data. In the presence of noise, a similar observation is made for large enough signal to noise ratio as in the $1 \mathrm{~mm}$ plate case. This error source can be mitigated with a finite element correction procedure. For this approach, an initial reconstructed pressure distribution is used as input for the numerical model described above. In practice, this is the experimentally identified distribution from the VFM. Processing the resulting slope maps obtained using the finite element model (see "Finite Element Model") yields the first iterated pressure distribution. The difference between this iteration and the original pressure reconstruction corresponds to the systematic error at every point of the pressure map. This difference is generally lower in amplitude than that between the original reconstruction and the real pressure distribution caused by systematic error, but it serves as a first estimation of that difference. Adding this difference to the original reconstruction yields an updated approximation of the real pressure distribution:

$d p_{\text {update }, \mathrm{n}}=p_{\text {rec }}+\left(p_{\text {rec }}-p_{\text {it, } \mathrm{n}}\right)$

This procedure can be repeated until $\left(p_{\text {rec }}-p_{\text {it,n }}\right)$ falls below a chosen threshold. Figure 14 shows how the input load is well recovered after only few iterations for modelled, noise free data. For the shown case, the second iteration result is already well converged and much closer to the input distribution, with an improvement from ca. $15 \%$ average error to below $6 \%$. Similar results were found for the other investigated PRW sizes.

An application to experimental data is more challenging. Each iteration tends to amplify noise patterns in pressure maps from both random and systematic error sources. Reconstructions from smoothed slope maps mitigate this issue, but suffer from a reduced number of available data points. Note that for each iteration, the size of one smoothing window, i.e. $6 \sigma_{\alpha}$, plus half a PRW of data points is lost around the edges (see also "Data Acquisition and Processing"). Here, this can be mitigated by using reconstructions with small slope smoothing kernels and by calculating circumferential averages from the stagnation point outwards, thus averaging out some of the random noise. These are then extrapolated to 2D distributions to obtain a suitable input for the finite element updating procedure. The entire process is applied to both numerical and experimental data, allowing
Fig. 15 Error estimates for circumferentially averaged pressure reconstructions for varying slope filter kernel and PRW size for $1 \mathrm{~mm}$ plate test and with grey level noise $0.95 \%$ of the dynamic range

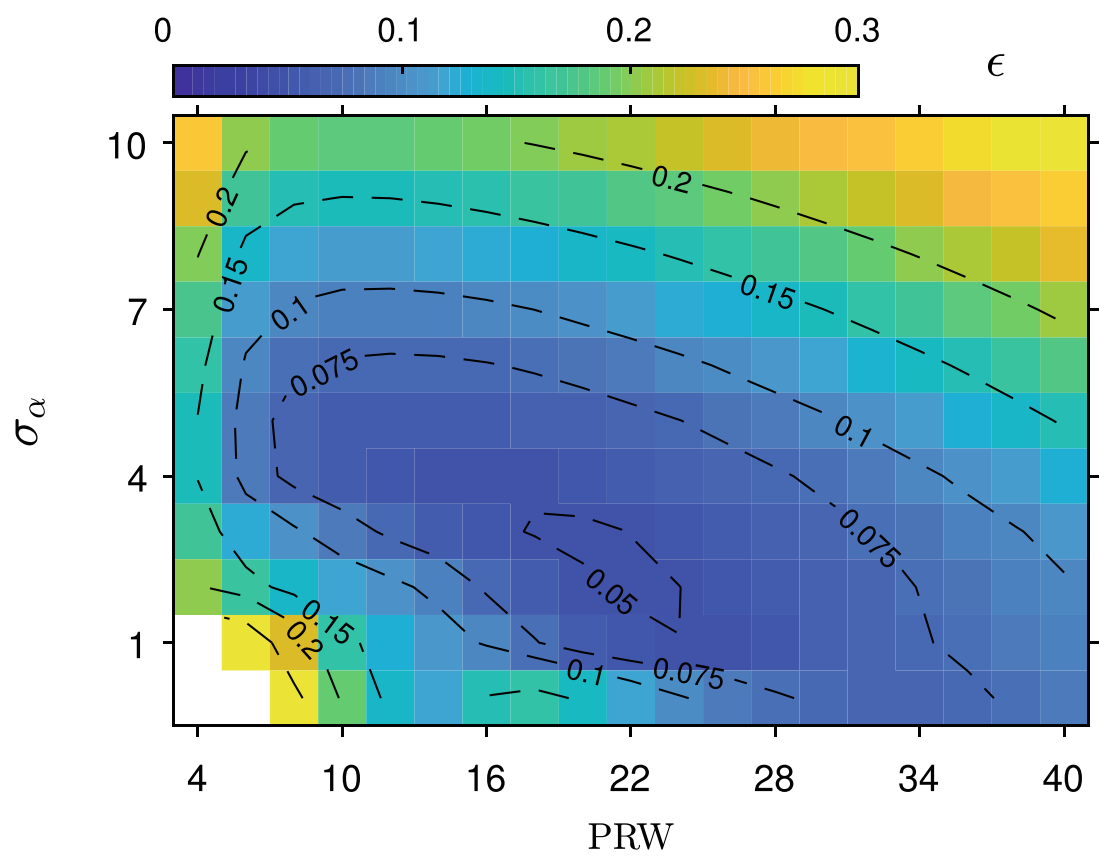


for a comparison of the results and thus further assessment of the influence of systematic experimental errors.

To select the correct reconstruction parameters for this approach, the accuracy assessment was repeated using circumferential averages instead of the entire field of view. The results vary, because low amplitude pressures are now averaged over a larger number of data points. Further, part of the field of view with low pressure amplitude is not taken into account as it is rectangular. The result is shown in Fig. 15. Figure 16 shows the results for iterations of experimental data and noisy model data. A $10 \%$ error bar corresponding to the estimated uncertainty resulting from the material's Young's modulus is shown for the iterations on experimental data at the positions of transducers for comparison. Figure 16(a) shows that for $\sigma_{\alpha}=3$ and PRW $=28$ the peak amplitude from transducer measurements is approximated to about $10 \%$ after 2 iterations of the experimental data. Since slope smoothing leads to a significant loss in data points, no further iterations are possible for this case. The corresponding numerical case, see Fig. 16(b), shows a close approximation of the input load.

For experimental data and $\sigma_{\alpha}=0$ and PRW $=34$, see Fig. 16(c), the influence of noise patterns becomes visible. These patterns are amplified by the correction procedure. Numerical data show a very good approximation of the input load, whereas experimental VFM data deviate from transducer data by ca. $10 \%$ after correction.

For $\sigma_{\alpha}=0$ and PRW $=22$, see Fig. 16(e), noise effects in experimental data are significant. Therefore, regularisation is necessary before iterating the results. Here, a fourth order polynomial was fitted to the averaged results. The iterated corrections once again approximate the transducer data
Fig. 16 Finite element updating results. Error bars on VFM represent the estimated uncertainty resulting from the material's Young's modulus. Error bars on transducer data represent both the systematic errors of the equipment as well as the random error of the mean pressure value

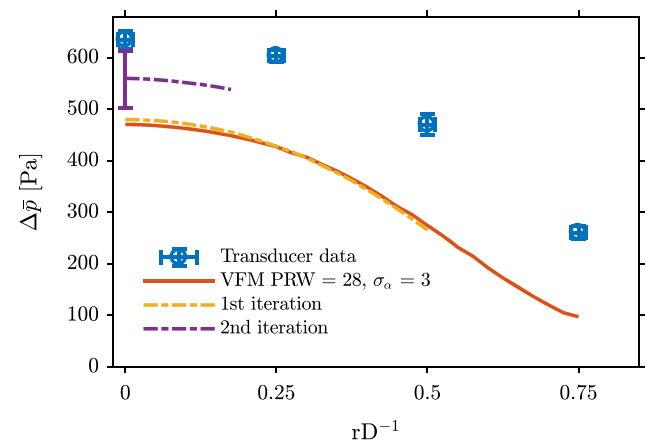

(a) Iteration of experimental data, $\sigma_{\alpha}=3$ and PRW $=28$.

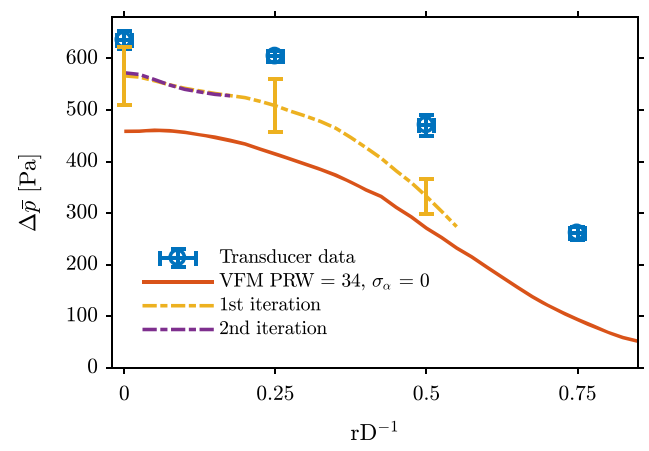

(c) Iteration of experimental data, $\sigma_{\alpha}=0$ and PRW $=34$.

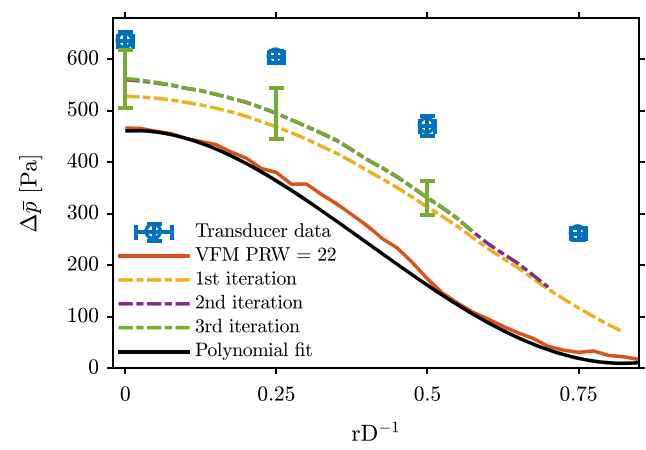

(e) Iteration of experimental data, $\sigma_{\alpha}=0$ and $\mathrm{PRW}=22$.

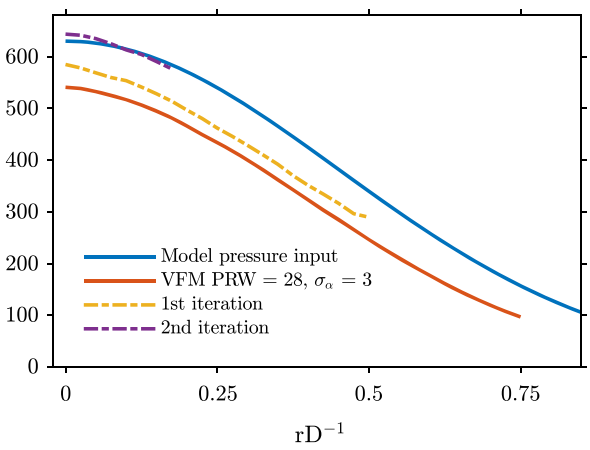

(b) Iteration of model data with noise, $\sigma_{\alpha}=3$ and PRW $=28$.

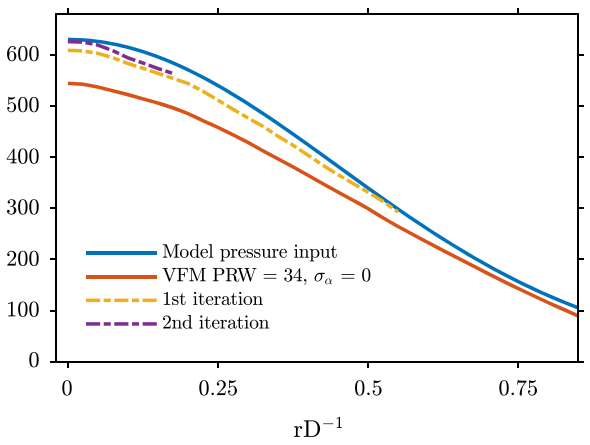

(d) Iteration of model data with noise, $\sigma_{\alpha}=0$ and PRW $=34$.

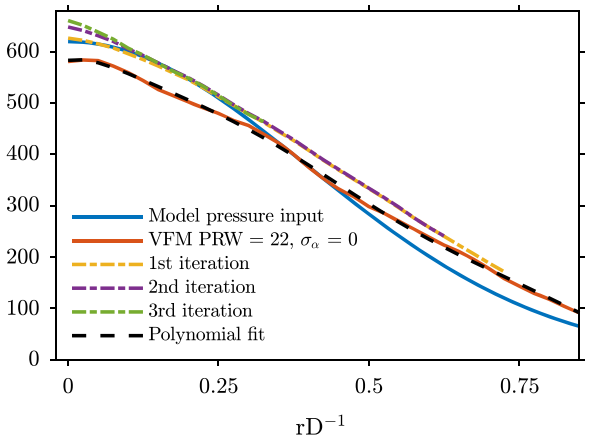

(f) Iteration of model data with noise, $\sigma_{\alpha}=0$ and PRW $=22$. 
to within ca. $10 \%$ of the peak amplitude. Figure 16(f) shows that for noisy model data an acceptable original estimation of the input amplitude is obtained. The corresponding corrected pressure distribution overestimates the peak and low range pressure amplitudes of the input distribution by ca. $5 \%$ of the peak amplitude. The in comparison to numerical data more pronounced noise patterns in experimental data (see also Figs. 11(b) and 12) were found to stem not only from random but also from systematic error sources (see "Experimental Results"). They may also be the reason for the large difference between experimental and numerical data in the initial reconstruction amplitude, here for $\mathrm{PRW}=22$ ca. $15 \%$.

All iterations appear reasonably well converged after the second iteration. Notably, the difference in peak amplitude is reduced to around $10 \%$ or better for all investigated cases. The outcome depends on the prevalence of noise patterns, which is more pronounced for small PRWs and small or no slope filters. However, larger reconstruction windows and filter kernels do not allow for many iterations since the loss of data points around the edges increases with PRW size.

\section{Error Sources}

The presented comparisons between real and simulated experiments have shown the influence of random noise and processing parameters on the pressure reconstruction. Experimental random noise patterns were qualitatively reproduced with the modelled data for all investigated cases. The presence of random noise was found to have a significant impact on the reconstruction results. A systematic error in the processing method was found to result in an underestimation of pressure amplitudes for noise-free model data. This error varies with the processing parameters. Further, a systematic experimental error appears between reconstructed and transducer-measured pressures. It was found that reconstructions from model data were consistently closer to the input data than the experimental reconstructions were to pressure transducer data, which are an established measurement technique. Based on the comparisons of numerical and experimental data shown in "Finite Element Correction", this error resulted in an additional underestimation of approximately $10 \%$ of the peak amplitude.

There are several possible sources for this experimental error. Miscalibration, i.e. non-integer numbers of pixels per pitch in the recorded grid, can lead to errors in the detected phases. It can be caused by misalignments between camera sensor and printed grid. Even with careful arrangement, small deformations of the specimen surface can cause misalignment issues. Note that these can also occur due to the deformations of the specimen under the investigated (dynamic) load. Misalignment can particularly result in fringes which can lead to the unexpected patterns observed in curvature maps in "Experimental Results". Irregularities and damages in the printed grid can also result in errors during phase detection. The influence of these error sources on pressure amplitude is however difficult to quantify. Another possible error source is wrong material parameter values, particularly the Young's modulus. The data information provided by the manufacturer gives a value of $E=74 \mathrm{GPa}$, but values between 47 and $83 \mathrm{GPa}$ are found for glass in the literature (e.g. [46, table 15.3]). 3- and 4-point bending tests on the specimen yielded values between 69 and $83 \mathrm{GPa}$ before the sample broke. Note that the relationship between Young's modulus and plate stiffness matrix components, and thus pressure amplitudes (see equation (4)), is linear, i.e. a $10 \%$ higher value of $E$ would increase all pressure amplitudes by $10 \%$, compensating for the discrepancy observed here. Deviations of the Poisson's ratio from the manufacturer information would have a similar impact. Since the plate stiffness matrix components are proportional to the third power of the plate thickness, errors in its determination have a higher impact than is the case for the other material parameters. Several measurements did however confirm the thickness values provided by the manufacturer. Assuming an error of $0.1 \%$ in the plate thickness as worst case estimate, one obtains a $3 \%$ error in the pressure amplitude.

Also, the assumptions of negligibility of rigid body movement and out of plane displacement need to be considered. LDV measurements on the frame holding the specimen showed no results above noise level, which corresponds to $0.1 \mu \mathrm{m}$ here. Rigid body movement can therefore be ruled out as a relevant error source. The effect of out of plane displacements can be estimated based on the expected deflections, w, and the distance between grid and specimen. A detailed derivation of this relationship is given in [43, chapter 2.1.2]. The resulting error on curvature maps is $\kappa_{\text {oop }}=\frac{w}{h_{\mathrm{S}}}$. The finite element simulations from "Simulated Experiments" showed that the deflections for the $1 \mathrm{~mm}$ plate test can be expected to be smaller than $2 \mu \mathrm{m}$, which would correspond to an error in curvature of $\kappa_{o o p}=210^{-3} \mathrm{~km}^{-1}$. This worst-case estimate corresponds to an error of only $0.05 \%$ of the peak curvature signal amplitude. Finally, the thin plate assumptions were tested using the finite element simulation introduced in "Finite Element Model". The chosen SHELL181 elements are suited for linear as well as for large rotation and large strain nonlinear applications. This means that simulated slopes and curvatures could deviate from those calculated from the deflections using thin plate assumptions (see e.g., [10]), if the latter were in fact not applicable. The simulated and the calculated slopes and curvatures were compared to verify the validity of the assumptions. For the $1 \mathrm{~mm}$ thick plate it was found that the difference was five orders of magnitude below the signal 
amplitude in case of slopes and thee orders of magnitude in case of curvatures.

\section{Limitations and Future Work}

This study shows that it is possible to obtain full-field pressure measurements of the order of few $\mathcal{O}(100) \mathrm{Pa}$ amplitude with the described setup and processing technique. A number of experimental limitations were encountered from applying this method to low amplitude loads. Small grid pitches are required to provide the required slope resolution. These require a very smooth and plane specular reflective specimen surface. Further decreasing the grid pitch would require more camera pixels to investigate the same region of interest, as the phase detection algorithm requires a minimum amount of pixels per pitch. Alternatively, the distance between grid and sample could be increased, which would require a different lens to achieve the same magnification. Furthermore, the specimen has to be stiff enough to provide a plane surface when mounted to avoid bias errors, but is required to deform sufficiently to provide enough signal for the measurement technique. The issue of misalignment could be addressed by using high precision components like micro stages with stepper motors to arrange camera, sample and grid.

Another approach is the use of infrared instead of visible light for deflectometry, with heated grids as spatial carrier [47]. Since infrared light has a longer wavelength than visible light, it allows achieving specular reflection on specimens that do not have mirror-like but reasonably smooth surfaces with up to about $1.5 \mu \mathrm{m}$ of RMS roughness, like perspex and metal plates. However, available cameras are limited in terms of spatial and temporal resolutions. Further issues are the lack of an aperture ring and that the lenses required to achieve comparable magnification are more expensive. An extension of the application of deflectometry to moderately curved surfaces was presented recently [34]. This approach requires a calibration for deformation measurement. Furthermore, the required depth of field is a restricting factor for the use of small grid pitches. A successful combination of deflectometry measurements on curved surfaces with VFM pressure reconstruction would be of great value, as it would allow direct measurements on practically relevant surfaces like e.g. aerofoils, fuselages and ship hulls.

In future studies, the turbulent fluctuations that occur in many practical flows like the impinging jet used here will be investigated. Typically they have pressure amplitudes of the order of few $\mathcal{O}(10) \mathrm{Pa}$ and below. These could not be resolved in this study. Preliminary analyses of time resolved data taken at $4 \mathrm{kHz}$ show that this is in parts due to a systematic experimental error, which results in spatial distributions fluctuating at low frequency and relatively high amplitude. The application of Fourier analyses and Dynamic Mode Decomposition (DMD) are currently being investigated with promising first results. Dynamic fullfield pressure reconstruction of turbulent fluctuations are a continuous challenge for current experimental measurement techniques due to their low amplitudes and small spatial scales, rendering the further development of the technique presented here highly relevant.

Another currently investigated improvement involves employing the aforementioned higher resolution cameras and smaller grid pitches to increase slope sensitivity and spatial resolution. This approach does not allow for time resolved measurements due to frame rate limitations of high resolution cameras, but first tests using phase averaging for periodic flows generated by synthetic jets are very promising.

Finally, the selection of virtual fields is an important factor in improving the quality of reconstructions. Particularly higher order approaches in pressure identification are likely to reduce the systematic error.

\section{Conclusion}

This work presents a method for surface pressure reconstructions from slope measurements using a deflectometry setup combined with the VFM. Experimental and numerical methods have been introduced to assess the pressure reconstructions.

- Low amplitude pressure distributions were reconstructed from full-field slope measurements using the material constitutive mechanical parameters.

- Experimental results are presented and compared for several reconstruction parameters and for two different specimen.

- VFM pressure reconstructions were compared to pressure transducer measurements.

- Simulated experiments employing a finite element model and artificial grid deformation were used to assess the uncertainty of the method.

- The numerical results were used to select optimal reconstruction parameters, taking into account experimentally observed noise.

- A finite element correction procedure was proposed to mitigate the systematic error of VFM pressure reconstructions.

- Error sources were discussed based on the findings of both the experimental and the simulated results.

A systematic processing error leading to an underestimation of the pressure amplitude was identified. Since the shape of the distribution is still reconstructed well, it is possible 
to compensate for this error using the proposed numerical approaches as long as noise patterns are not too pronounced. A systematic experimental error was found to result in an additional underestimation of the pressure amplitude by ca. $10 \%$ more than simulated reconstructions. Yet, the results stand out in terms of the low pressure amplitudes and the large number of data points obtained.

\section{Data Provision}

All relevant data produced in this study is available under the DOI https://doi.org/10.5258/SOTON/D0973.

Acknowledgements This work was funded by the Engineering and Physical Sciences Research Council (EPSRC). F. Pierron acknowledges support from the Wolfson Foundation through a Royal Society Wolfson Research Merit Award (2012-2017). Advice and assistance given by Cédric Devivier, Yves Surrel, Manuel Aguiar Ferreira and Lloyd Fletcher has been a great help in conducting simulations and planning of experiments. The comments provided by Manuel Aguiar Ferreira and Lloyd Fletcher have greatly improved this paper.

Open Access This article is distributed under the terms of the Creative Commons Attribution 4.0 International License (http:// creativecommons.org/licenses/by/4.0/), which permits unrestricted use, distribution, and reproduction in any medium, provided you give appropriate credit to the original author(s) and the source, provide a link to the Creative Commons license, and indicate if changes were made.

\section{References}

1. Usherwood JR (2009) The aerodynamic forces and pressure distribution of a revolving pigeon wing. Exp Fluids 46(5):9911003

2. Livingood NBJ, Hrycak, P (1973) Impingement heat transfer from turbulent air jets to flat plates: A literature survey. Tech. Rep. NASA-TM-x-2778, E-7298, NASA Lewis Research Center; Cleveland, $\mathrm{OH}$

3. Corcos GM (1963) Resolution of pressure in turbulence. J Acoust Soc Amer 35(2):192-199

4. Corcos GM (1964) The structure of the turbulent pressure field in boundary-layer flows. J Fluid Mech 18(3):353-378

5. Tropea C, Yarin A, Foss J (2007) Springer handbook of experimental fluid mechanics. Springer, Berlin

6. Yang L, Zare-Behtash H, Erdem E, Kontis K (2012) Application of AA-PSP to hypersonic flows: The double ramp model. Sens Actuators B: Chem 161(1):100-107

7. van Oudheusden BW (2013) PIV-based pressure measurement. Measur Sci Technol 032(3):001

8. Ragni D, Ashok A, van Oudheusden BW, Scarano F (2009) Surface pressure and aerodynamic loads determination of a transonic airfoil based on particle image velocimetry. Measur Sci Technol 20(7):074,005

9. Brown K, Brown J, Patil M, Devenport W (2018) Inverse measurement of wall pressure field in flexible-wall wind tunnels using global wall deformation data. Exp Fluids 59(2):25
10. Timoshenko S, Woinowsky-Krieger S (1959) Theory of plates and shells. Engineering societies monographs. McGraw-Hill, New York

11. Pezerat C, Guyader JL (2000) Force Analysis Technique: Reconstruction of force distribution on plates. Acta Acust United Acust 86(2):322-332

12. Leclére Q, Pézerat C (2012) Vibration source identification using corrected finite difference schemes. J Sound Vibr 331(6):13661377

13. Lecoq D, Pézerat C, Thomas JH, Bi W (2014) Extraction of the acoustic component of a turbulent flow exciting a plate by inverting the vibration problem. J Sound Vibr 333(12):25052519

14. Pierron F, Grédiac M (2012) The virtual fields method. Extracting constitutive mechanical parameters from full-field deformation measurements. Springer, New York

15. Martins J, Andrade-Campos A, Thuillier S (2018) Comparison of inverse identification strategies for constitutive mechanical models using full-field measurements. Int J Mech Sci 145:330-345

16. Moulart R, Pierron F, Hallett SR, Wisnom MR (2011) Fullfield strain measurement and identification of composites moduli at high strain rate with the virtual fields method. Exp Mech 51(4):509-536

17. Pierron F, Sutton MA, Tiwari V (2011) ultra high speed DIC and virtual fields method analysis of a three point bending impact test on an aluminium bar. Exp Mech 51(4):537-563

18. Robin O, Berry A (2018) Estimating the sound transmission loss of a single partition using vibration measurements. Appl Acoust 141:301-306

19. Berry A, Robin O, Pierron F (2014) Identification of dynamic loading on a bending plate using the virtual fields method. J Sound Vibr 333(26):7151-7164

20. Toussaint E, Grédiac M, Pierron F (2006) The virtual fields method with piecewise virtual fields. Int J Mech Sci 48(3):256264

21. Berry A, Robin O (2016) Identification of spatially correlated excitations on a bending plate using the virtual fields method. J Sound Vibr 375:76-91

22. O'Donoughue P, Robin O, Berry A (2017) Time-resolved identification of mechanical loadings on plates using the virtual fields method and deflectometry measurements. Strain 54(3):e12,258

23. Surrel Y, Fournier N, Grédiac M, Paris PA (1999) Phase-stepped deflectometry applied to shape measurement of bent plates. Exp Mech 39(1):66-70

24. Devivier C, Pierron F, Wisnom M (2012) Damage detection in composite materials using deflectometry, a full-field slope measurement technique. Compos Part A: Appl Sci Manuf 43(10):1650-1666

25. Giraudeau A, Pierron F, Guo B (2010) An alternative to modal analysis for material stiffness and damping identification from vibrating plates. J Sound Vib 329(10):1653-1672

26. Devivier C, Pierron F, Glynne-Jones P, Hill M (2016) Timeresolved full-field imaging of ultrasonic Lamb waves using deflectometry. Exp Mech 56:1-13

27. O'Donoughue P, Robin O, Berry A (2019) Inference of random excitations from contactless vibration measurements on a panel or membrane using the virtual fields method. In: Ciappi E, De Rosa S, Franco F, Guyader JL, Hambric SA, Leung RCK, Hanford $\mathrm{AD}$ (eds) Flinovia-flow induced noise and vibration issues and aspects-II. Springer International Publishing, Cham, pp 357372

28. Kalifa RB, Habli S, Saïd NM, Bournot H, Palec GL (2016) The effect of coflows on a turbulent jet impacting on a plate. Appl Math Modell 40(11):5942-5963 
29. Zuckerman N, Lior N (2006) Jet impingement heat transfer: physics, correlations, and numerical modeling. Adv Heat Transfer 39(C):565-631

30. Beltaos S (1976) Oblique impingement of circular turbulent jets. J Hydraul Res 14(1):17-36

31. Grédiac M, Sur F, Blaysat B (2016) The grid method for inplane displacement and strain measurement: a review and analysis. Strain 52(3):205-243

32. Ritter R (1982) Reflection moire methods for plate bending studies. Opt Eng 21:21-29

33. Balzer J, Werling S (2010) Principles of shape from specular reflection. Measurement 43(10):1305-1317

34. Surrel Y, Pierron F (2019) Deflectometry on curved surfaces. In: Proceedings of the 2018 Annual Conference on Experimental and Applied Mechanics, pp 217-221

35. Dai X, Xie H, Wang Q (2014) Geometric phase analysis based on the windowed Fourier transform for the deformation field measurement. Opt Laser Technol 58:119-127

36. Surrel Y (2000) Photomechanics, chap. Fringe Analysis. Springer, Berlin, pp 55-102

37. Poon CY, Kujawinska M, Ruiz C (1993) Spatial-carrier phase shifting method of fringe analysis for moiré interferometry. J Strain Anal Eng Des 28(2):79-88

38. Surrel Y (1996) Design of algorithms for phase measurements by the use of phase stepping. Appl Opt 35(1):51-60

39. Hibino K, Larkin K, Oreb B, Farrant D (1995) Phase shifting for nonsinusoidal waveforms with phase-shift errors. J Opt Soc Am A 12(4):761-768
40. Badulescu C, Grédiac M, Mathias JD (2009) Investigation of the grid method for accurate in-plane strain measurement. Measur. Sci. Technol 20(9):095,102

41. Dym C, Shames I (1973) Solid mechanics: a variational approach. Advanced engineering series. McGraw-Hill, New York

42. Zienkiewicz O (1977) The finite element method. McGraw-Hill, New York

43. Devivier C (2012) Damage identification in layered composite plates using kinematic full-field measurements. Ph.D. thesis Université de Technologie de Troyes

44. Barbero E (2013) Finite element analysis of composite materials using ANSYS $®$, 2nd edn. Composite Materials. CRC Press, Boca Raton

45. Rossi M, Pierron F (2012) On the use of simulated experiments in designing tests for material characterization from full-field measurements. Int J Solids Struct 49(3):420-435

46. Ashby M (2011) Materials selection in mechanical design, 4th edn. Butterworth-Heinemann, Oxford

47. Toniuc H, Pierron F (2018) Infrared deflectometry for surface slope deformation measurements Experimental Mechanics. (submitted)

Publisher's Note Springer Nature remains neutral with regard to jurisdictional claims in published maps and institutional affiliations. 